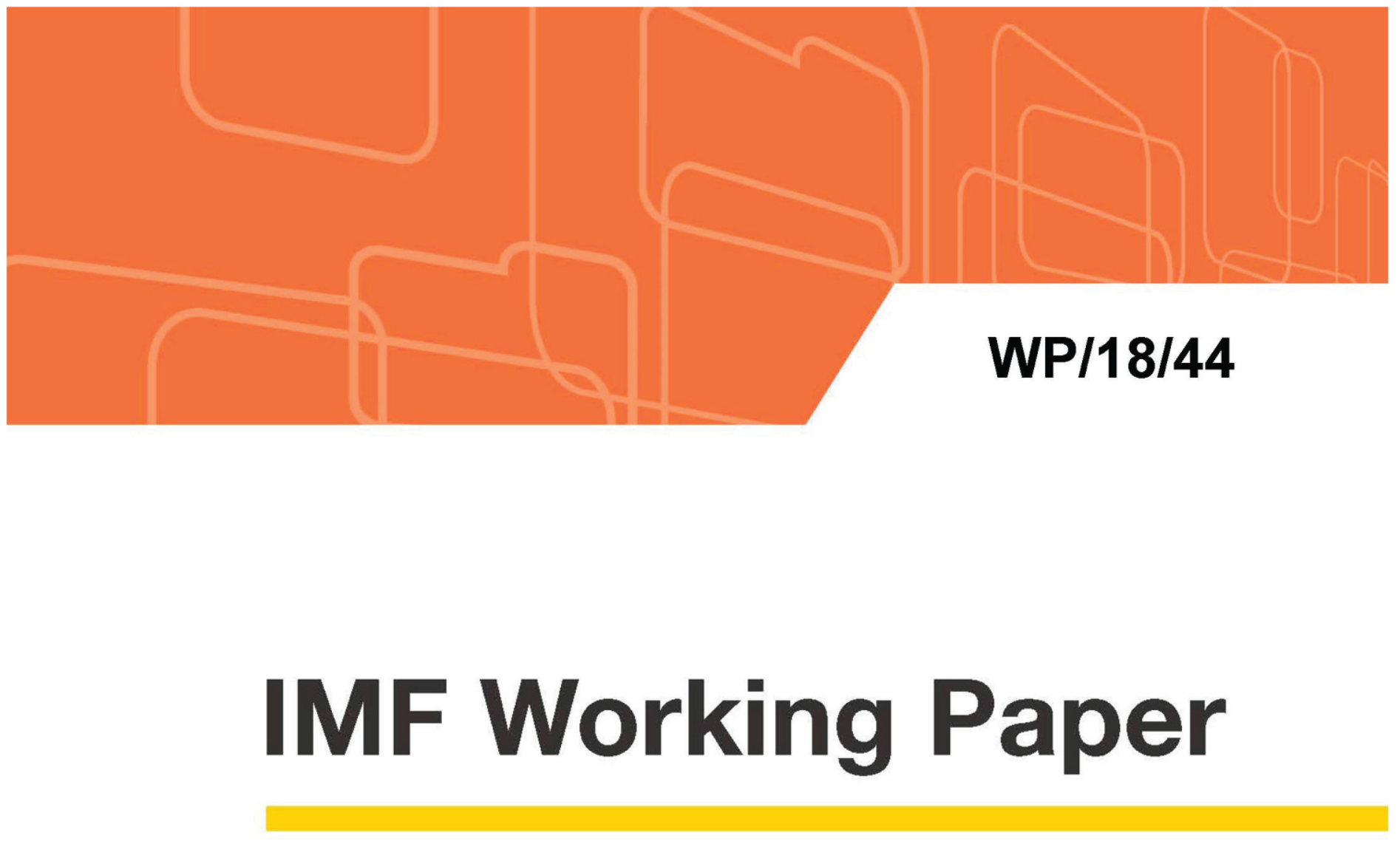

\title{
Is There a Phillips Curve? A Full Information Partial Equilibrium Approach
}

\author{
by Roberto Piazza
}

IMF Working Papers describe research in progress by the author(s) and are published to elicit comments and to encourage debate. The views expressed in IMF Working Papers are those of the author(s) and do not necessarily represent the views of the IMF, its Executive Board, or IMF management. 


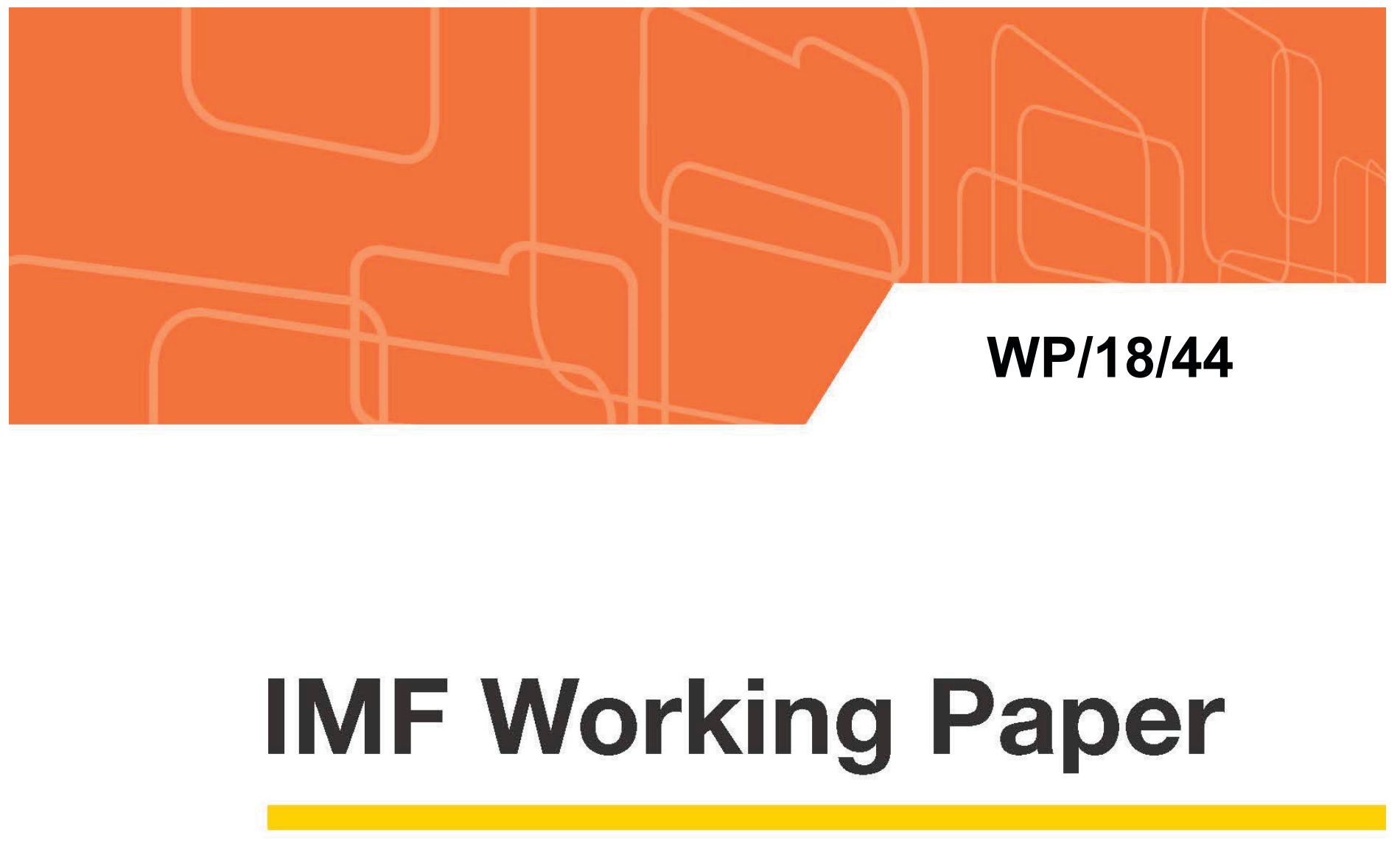

\section{Is There a Phillips Curve? A Full Information Partial Equilibrium Approach}

by Roberto Piazza

IMF Working Papers describe research in progress by the author(s) and are published to elicit comments and to encourage debate. The views expressed in IMF Working Papers are those of the author(s) and do not necessarily represent the views of the IMF, its Executive Board, or IMF management.

$$
\text { I N T E R N A T I O N A L M O N E T A R Y F U N D }
$$


IMF Working Paper

\title{
Is There a Phillips Curve? A Full Information Partial Equilibrium Approach
}

by Roberto Piazza

Authorized for distribution by Helge Berger

March 2018

\section{IMF Working Papers describe research in progress by the author(s) and are published to elicit comments and to encourage debate. The views expressed in IMF Working Papers are those of the author(s) and do not necessarily represent the views of the IMF, its Executive Board, or IMF management.}

\begin{abstract}
Empirical tests of the New Keynesian Phillips Curve have provided results often inconsistent with microeconomic evidence. To overcome the pitfalls of standard estimations on aggregate data, a Full Information Partial Equilibrium approach is developed to exploit sectoral level data. A model featuring sectoral NKPCs subject to a rich set of shocks is constructed. Necessary and sufficient conditions on the structural parameters are provided to allow sectoral idiosyncratic components to be linearly extracted. Estimation biases are corrected using the model's restrictions on the partial equilibrium propagation of idiosyncratic shocks. An application to the US, Japan and the UK rejects the purely forward looking, labor cost-based NKPC.
\end{abstract}

JEL Classification Numbers: E30, E310, E370

Keywords: Phillips Curve, Full Information, Limited Information, sticky prices

Author's E-Mail Address: rpiazza2@imf.og

\footnotetext{
* This paper builds on an earlier unpublished work with A. Anzuini. I thank Alessio for the comments provided for this new paper. I also thank John Roberts and Luigi Paciello for insightful suggestions. The views expressed here are those of the author and do not necessarily represent the views of the IMF. All errors are my own.
} 
Abstract …

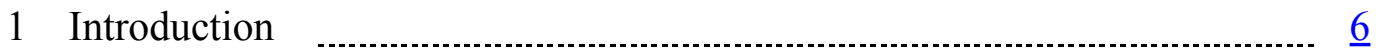

2 Sectoral Phillips curves in a standard New Keynesian model …................. 9

2.1 Preferences and production technologies …_........................

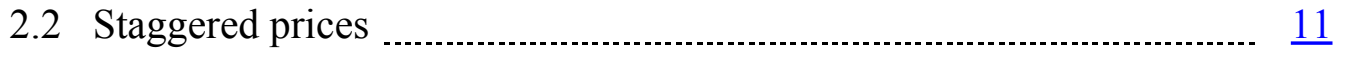

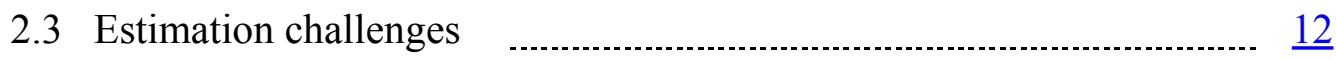

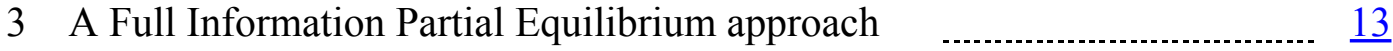

3.1 Separating aggregate and idiosyncratic components $\ldots \ldots \ldots \ldots \ldots \ldots \ldots \ldots \ldots$

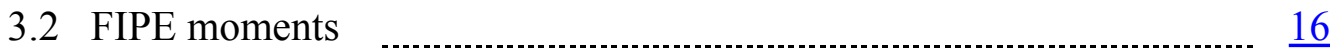

3.3 Beyond the pure forward-looking model …............................... 18

4 FIPE in a Bayesian application $\ldots \ldots \ldots$

4.1 An ARMA(1,1) specification ……………….............................. 19

4.2 Data description and priors ………………….............................. 21

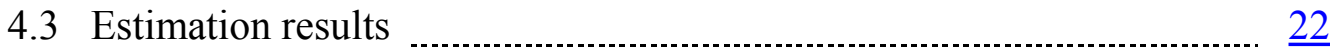

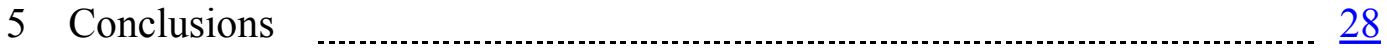

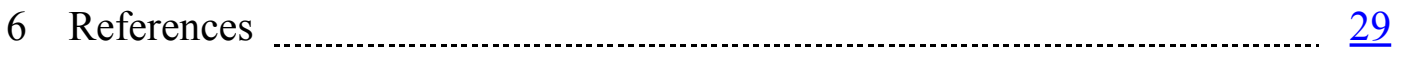

Appendices

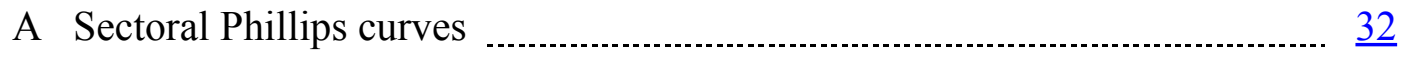

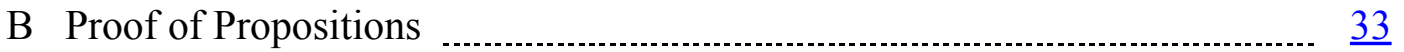

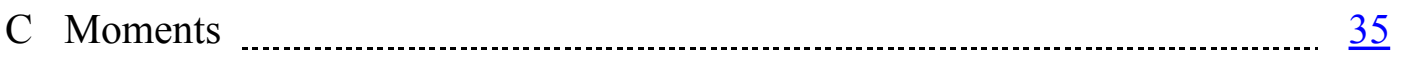

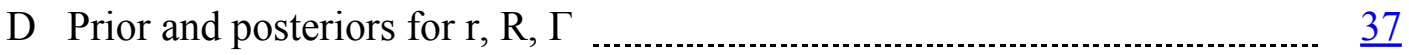

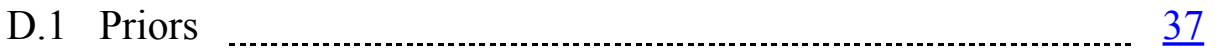




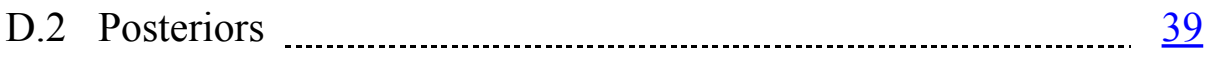

D.3 Posteriors diagnostics ………………..........................

Figures

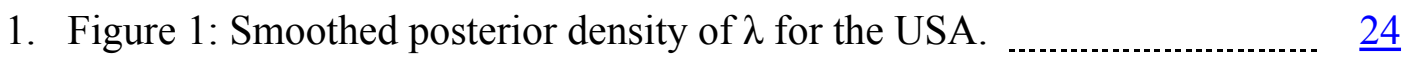

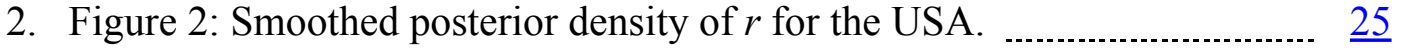

3. Figure 3: Smoothed posterior density of $\lambda$ for Japan. ............................. $\underline{26}$

4. Figure 4: Smoothed posterior density of $\lambda$ for the UK. ......................... 27

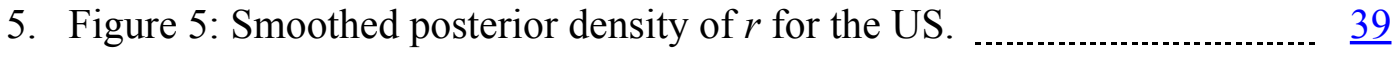

6. Figure 6: Smoothed posterior density of $R$ for the US. ...........................

7. Figure 7: Smoothed posterior density of $\Gamma$ for the US. ........................... $\underline{41}$

8. Figure 8: Smoothed posterior density of $r$ for Japan. ............................. $\underline{42}$

9. Figure 9: Smoothed posterior density of $R$ for Japan.

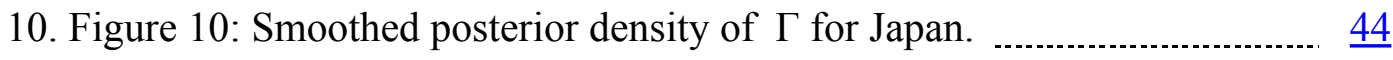

11. Figure 11: Smoothed posterior density of $r$ for the UK. ....................... $\underline{45}$

12. Figure 12: Smoothed posterior density of $R$ for the UK. ...................... $\underline{46}$

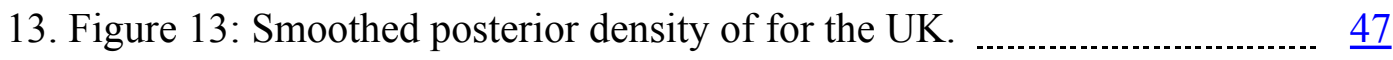

14. Figure 14: Auto-correlation function of draws for $\lambda$ and the US. .............. $\underline{48}$

15. Figure 15: Auto-correlation function of draws for $r$ and the US. ............... $\underline{49}$

16. Figure 16: Auto-correlation function of draws for $R$ and the US. .............. $\underline{50}$

17. Figure 17: Auto-correlation function of draws for $\Gamma$ and the US. ............ $\underline{51}$

18. Figure 18: Auto-correlation function of draws for $\lambda$ and Japan. ............... $\underline{52}$

19. Figure 19: Auto-correlation function of draws for $r$ and Japan. ................. $\underline{53}$

20. Figure 20: Auto-correlation function of draws for $R$ and Japan. ............... $\underline{54}$

21. Figure 21: Auto-correlation function of draws for $\Gamma$ and Japan. ................ $\underline{55}$ 
22. Figure 22: Auto-correlation function of draws for $\lambda$ and the UK.

23. Figure 23: Auto-correlation function of draws for $r$ and the UK.

24. Figure 24: Auto-correlation function of draws for $R$ and the UK. $\underline{58}$

25. Figure 25: Auto-correlation function of draws for $\Gamma$ and the UK. $\underline{59}$ 


\section{Introduction}

In its standard formulation, the New Keynesian Phillips Curve (NKPC) relates current inflation $\pi_{t}$ to the current gap $\hat{x}_{t}$ of a real forcing variable (the "real marginal cost gap" or the "output gap"), to the long-run level $\bar{\pi}_{t}$ of inflation (commonly interpreted as a time-varying inflation target) and to the expected value $E_{t} \pi_{t+1}^{i}$ of next period's inflation. The forward-looking nature of the NKPC is a pivotal feature heavily exploited for its policy implications.

Considerable effort has been dedicated to the estimation of the slope of the NKPC, i.e. the parameter $\lambda$ in front of $\hat{x}_{t}$. However, estimating the parameters of the NKPC is not an easy task. Major econometric concerns arise for mainly two reasons. First, the forcing variable gap $\hat{x}_{t}$ and the long-run inflation $\bar{\pi}_{t}$ are not observable. In their place, proxies can be used, but this introduces errors that are likely endogenous and can thus lead to biased estimates. Second, the unobserved long-run inflation $\bar{\pi}_{t}$ is non-stationary over sample periods characterized by switches in the monetary policy regime. Hence, the corresponding omitted variable errors are also non-stationary. Estimating the NKPC in first differences could in principle solve the non-stationarity problem, but unfortunately it would also worsen error endogeneity concerns, by introducing correlation between measurement error about inflation expectations at $t-1$ and the current value of $\hat{x}_{t}$.

The literature has taken two main approaches to tackle the error endogeneity problem. The first is based on single-equation Limited Information (LI) techniques. The guiding principle is to estimate the NKPC by applying GMM/IV methods to correct for the potential estimation bias. Mavroedis et al. (2014) present an extensive survey of this literature, which features a wide set of point estimates, often suffering from weak identification. ${ }^{1}$ The second is the Full Information (FI) approach, where the NKPC is estimated jointly with all the other structural equations of the NK model. The joint estimation aims to tackle the endogeneity problem head-on by explicitly characterizing the endogenous interactions of the macroeconomic variables through the restrictions derived from the fully-specied NK model. Schorfheide (2008) surveys this part of the literature and finds, again, a wide range of empirical estimates for $\lambda$, a significant fraction of which appears too small - namely they imply too much price stickiness - to be consistent with the degree of price rigidity that emerges from microeconomic evidence (Nakamura and Steinsson 2013). Overall, both LI and FI methods have strengths and weaknesses. The former is less prone to model misspecification, but the use of instruments is subject to problems of weak of identification and lack of robustness. The latter, while addressing the endogeneity issue in a more direct and coherent way, delivers in reality a test on the validity of the entire NK model, and not just of the NKPC.

In light of the surveyed evidence, Mavroedis et a. (2014) conclude that "the literature has reached a limit in how much can be learned about the New Keyne-

\footnotetext{
${ }^{1}$ Seminal papers are Roberts (1995), Fuhrer and Moore (1995), Fuhrer (1997), Galí and Gertler (1999), Sbordone (2002), Rudd and Whelan (2004), Mavroedis (2004), Lindé (2005), Roberts (2005).
} 
sian Phillips curve from aggregate macroeconomic time series. New identification approaches and new datasets are needed to reach an empirical consensus".

The goal of this paper is to propose a new methodology for the identification of the NKPCs. I name it Full Information Partial Equilibrium (FIPE) to emphasize that it is intended to combine the strengths of both the FI and the LI approaches. FIPE estimations are designed to move away from aggregate time series, and instead be applied to the extensive sectoral datasets that, in recent years, have become available for many countries. The proposed methodology is very flexible along two important dimensions. First, while the paper illustrates the workings of a FIPE approach in the context of a purely forward-looking model, it also indicates how to adapt the methodology to estimate popular alternative ("hybrid") versions of the NKPC. Second, a FIPE analysis provides general closed-form statistics that can be exploited by a variety of econometric techniques, such as GMM or MLE methods. I present, as an example, an application in a Bayesian estimation setting.

The FIPE approach is centered around estimating sectoral NKPCs using, for identification, only the variability in sectoral gross output inflation and real marginal costs due to idiosyncratic shocks (demand, supply, mark-ups, long-run sectoral inflation). Similarly to aggregate shocks, idiosyncratic shocks cause endogeneity problems in the estimation of the NKPC. However, their propagation occurs in partial equilibrium and can thus be modeled explicitly by postulating minimal structural assumptions on sectoral demand and supply functions. Therefore, the FIPE strategy incorporates the FI intuition that estimation biases can be corrected by exploiting the restrictions from the model's equilibrium conditions. In addition, the FIPE approach mimics the robustness of LI estimations to potential misspecifications of general equilibrium effects. In this way, the advantages of FI and LI are leveraged within a single procedure. The structural specification can then deliver consistent estimates even where popular LI identification strategies fail, e.g. when mark-up shocks are auto-correlated or in the presence of non stationary errors. ${ }^{2}$ At the same time, contrary to FI estimations, the focus on partial equilibrium dynamics renders unnecessary the specifications of structural equations such as the monetary policy rule.

The proposed methodology delivers a set of statistics, called FIPE moments. These are represented by closed-form theoretical covariances of sectoral inflation rates and real marginal costs, expressed as functions of the deep parameters of the model. They include, among others, sectoral Phillips curve slopes and the exogenous auto-covariances of sectoral structural shocks. Some parameters may be directly calibrated. The remaining free parameters can instead be estimated by combining the theoretical moments with empirical observations.

As mentioned, the empirical data must represent time series variation of inflation and real marginal costs generated only by idiosyncratic shocks. As a preliminary step to estimation, the data must therefore be purged from the effect

\footnotetext{
${ }^{2}$ For example, LI estimations usually need to assume that the unobserved error terms, commonly interpreted as a "mark-up" shock, is uncorrelated across time. In fact, if the mark-up shocks were allowed to be auto-correlated, then lags of the endogenous variables typically used in IV estimations would not be valid instruments.
} 
of aggregate shocks. To this end, the paper provides necessary and sufficient conditions on sectoral parameters that allow the inflation and real marginal cost series to be linearly decomposed into two parts. One is the series variation caused by idiosyncratic shocks. The other is the component attributable to aggregate shocks, expressed as the product of common components and sector specific loadings. Aggregate shocks can then be preliminarily removed from the series by means of standard principal components procedures applied to a panel of sectors. Hence, the paper provides the theoretical conditions that justify the form of the inflation process postulated in Bovin et al. (2009).

An interesting issue connected to our empirical strategy is how sectoral slopes estimated from idiosyncratic shocks are related to the aggregate slope $\lambda$. With this regard, the theoretical literature has emphasized two main points. First, in the presence of strategic complementarities, the aggregate degree of Calvo price stickiness is disproportionally influenced by the most sticky among the cross section of sectors (Carvalho 2006). The second point deals instead with the within sector response of prices to shocks. Models with Calvo price adjustments, such as the one studied in this paper, are ill-suited to provide a justification for the presence of different frequencies of price adjustment to aggregate versus idiosyncratic shocks. As a consequence, in the Calvo model the slope of the sectoral Phillips curve is assumed to be the same in response to both shocks (Maćkowiak et al. 2009). However, models where stickiness is explicitly based on firms' rational inattention predict that prices should be more flexible in response to idiosyncratic shocks (Maćkowiak and Wiederholt 2009, Melosi 2014). Taken together, these considerations indicate that first moments $\bar{\lambda}$ (such as the median or the mean) of the cross section of sectoral FIPE estimates of Phillips curves slopes should be larger than the aggregate Phillips curve $\lambda$. Since they imply less price stickiness, summary statistics $\bar{\lambda}$ should then be easier to match with the micro evidence on the frequency of price adjustment.

As an application, I use FIPE moments in conjuction with Bayesian techniques to estimated sectoral NKPC slopes for two large economies, the US and Japan, and a small open economy, the UK. Real marginal costs are proxied by the labor share. The estimation results echo findings common in the empirical literature: first moments $\bar{\lambda}$ of the cross section of sectoral slopes have either the wrong sign or, when they are positive, their values appear to be too small to be reconciled with the degree of price rigidity inferred from the micro evidence (Nakamura and Steinsson 2008, Bils and Kryvtsov 2008, Kehoe an Midrigan 2015).

The rest of the paper is organized as follows. Section 2 lays out the assumption on sectoral demand and supply functions, and develops sectoral Phillips curves with time varying long-run inflation rates. Section 3 gives necessary and sufficient conditions for the linear decomposition of aggregate and idiosyncratic shocks, and derives the FIPE moments. Section 4 presents the results of the empirical application. Section 5 concludes. 


\section{Sectoral Phillips curves in a standard New Keynesian model}

This section derives a sectoral version of the standard NKPC,

$$
\pi_{t}^{i}-\bar{\pi}_{t}^{i}=\lambda_{i} \hat{m}_{t}^{r i}+\beta E_{t}\left[\pi_{t+1}^{i}-\bar{\pi}_{t+1}^{i}\right]
$$

where $\beta \in(0,1)$ is a discount factor, $\hat{m}_{t}^{r i}$ is the marginal cost gap, i. e. the forcing variable of the NKPC, $E_{t}\left[\pi_{t+1}^{i}\right]$ is the inflation expectation and $\bar{\pi}_{t}^{i}$ is a sector specific variable used as indexation by Calvo firms that cannot freely reset their prices at $t$. The semi-structural parameter $\lambda_{i}$, which is the slope of the Phillips curve, is a function of an underlying parameters $\theta_{i}$ defining the degree of price stickiness.

\subsection{Preferences and production technologies}

There is a double continuum of goods $(i, j)$ in the economy, with $i \in[0,1]$ representing a sector and $j \in[0,1]$ indicating a particular variety within sector $j$. Each pair $(i, j)$ represents both the good and the firm which produces it under monopolistic competition. The variables $\tilde{y}_{t}(i, j), y_{t}(i)$ and $Y_{t}$ indicate, respectively, the $\log$ output of good $(i, j)$, the $\log$ aggregate output of sector $i$, and the total log aggregate output of the economy at time $t$. Log prices are defined in the same fashion.

Demand functions for good $(i, j)$ and for sector $i$ 's overall bundle are derived from constant elasticity utility aggregators with elasticities given by $\nu_{t}^{i}$ and $\bar{\nu}$, respectively. Specifically, the consumer demand for good $(i, j)$ at time $t$ is given by

$$
\tilde{y}_{i}(i, j)=y_{t}(i)-\nu_{t}^{i}\left[\tilde{p}_{t}(i, j)-p_{t}(i)\right]
$$

where the elasticity $\nu_{t}^{i}>1$ is time varying. For future reference, we define $\tilde{\mu}_{t}^{i}$ as the log sectoral target mark-up

$$
\tilde{\mu}_{t}^{i}=\log \frac{\nu_{t}^{i}}{\nu_{t}^{i}-1}
$$

Similarly, sectoral demands relative to aggregate demand, written in first difference, are defined as

$$
\Delta y_{t}(i)=\Delta Y_{t}-\bar{\nu}\left[\pi_{t}^{i}-\pi_{t}-\Delta \omega_{t}^{i}\right]
$$

where $\pi_{t}^{i}=p_{t}(i)-p_{t-1}(i)$ and $\pi_{t}=P_{t}-P_{t-1}$ are, respectively, sectoral and aggregate inflation and $\bar{v}>1$. Moreover, $\omega_{t}^{i}>0$ is an exogenous sectoral demand shifter, presented in more details below.

We turn to the definition of firms' supply functions. We assume that the log nominal marginal cost is the same for all firms $j$ within a given sector and is given by

$$
m_{t}^{n i}=\sum_{k=1}^{K_{m}-1} l_{k i} \phi_{k t}+\frac{\delta_{i}}{\bar{\nu}} y_{t}(i)-\tilde{z}_{t}^{i}
$$


The variable $\tilde{z}_{t}^{i}$ is the log of the sectoral TFP and the parameter $\delta_{i}$ indicates production real rigidities at the sectoral level. We usually think of $\delta_{i}$ as positive, but negative values are also allowed, e.g. if there are decreasing marginal cost at the sectoral level. In turn, the sets of $K_{m}-1 \geq 1$ sector-specific constants $l_{k i}$ and variables $\phi_{k t}$ represent, respectively, sectoral loadings an aggregate factors which cause co-movements in sectoral log nominal marginal costs. By using the non-differenced version of (4) it is straightforward to derive the sectoral log real marginal cost $m_{t}^{r i} \equiv m_{t}^{n i}-p_{t}(i)$ as

$$
m_{t}^{r i}=\sum_{k=1}^{K_{m}} l_{k i} \phi_{k t}+\bar{\nu}^{-1}\left(1+\delta_{i}\right) y_{t}(i)-\tilde{z}_{t}^{i}-\omega_{t}^{i}
$$

where the $K_{m}$-th additional factor is $\phi_{K t}=-P_{t}-\bar{\nu}^{-1} Y_{t}$. Without loss of generality we take $\sum_{k}^{K_{m}} l_{k i}=1$. The form (6) for the log real marginal cost is a crucial equation of our model. It is important to point out that (6) is quite general and can be obtained, either directly or through log-linear approximations, from a variety of production functions. The constant $\delta_{i}$ controls the responsiveness of real marginal costs to output, as discussed in Rotemberg and Woodford (1999). The following example with Cobb-Douglas production function explains how increasing marginal costs at the sectoral level arise naturally, for instance, in the presence of fixed or quasi-fixed production factors.

Example. Normalize $\bar{\nu}=1$. Assume that, at the firm's level, the log production function is specified by $\tilde{y}_{t}(i, j)=Z_{t}+\tilde{z}_{t}^{i}+\tilde{n}_{t}(i, j)$, where $Z_{t}$ is aggregate log TFP and $n_{t}(i, j)$ is the labor input. Using an appropriate Dixit-Stiglitz function, we assume that firms' production in sector $i$ is aggregated into a sectoral output $y_{t}(i)$, which is given by $y_{t}(i)=Z_{t}+n_{t}(i)$, with $n_{t}(i)$ the corresponding sectoral aggregator for the labour input. The log labour supply schedule at the sectoral level is assumed to be $w_{t}^{i}=W_{t}+\delta_{i}\left[n_{t}(i)-N_{t}\right]$, with $w_{t}^{i}$ the sectorwide nominal $\log$ salary, while $W_{t}$ and $N_{t}$ are, respectively, the economy-wide nominal salary and employment. If we take $\delta_{i}>0$, then the labour supply schedule implies that higher than average employment in sector $i$ translates into higher than average sectoral salaries. Salaries may vary across sectors as function of labour utilization because of, for instance, overtime wage premia, labor market segmentation across sectors or the presence of labour unions. It is then straightforward to show that the nominal log marginal cost in sector $i$ is $m_{t}^{n i}=W_{t}-\delta_{i} N_{t}-\left(1+\delta_{i}\right) Z_{t}+\delta_{i} y_{t}(i)-\left(1+\delta_{i}\right) \tilde{z}_{t}^{i}$. After an appropriate scaling of $\tilde{z}_{t}^{i}$, we obtain a formulation of the type (5).

We conclude this section with the specification of the stochastic process for the exogenous variables of the model. In some instances, shocks are presented into a normalized form to yield less cluttered equilibrium conditions. The process for the sectoral demand shifter $\omega_{t}^{i}$ is the following,

$$
\omega_{t}^{i}=\rho_{\omega}^{i} \omega_{t-1}^{i}+\delta_{i}^{-1} \epsilon_{t}^{i \omega}
$$


The log sectoral mark-up $\hat{\mu}_{t}^{i}$ is assumed to be the sum of an aggregate component $\bar{\mu}_{t}$ and an $\operatorname{AR}(1)$ idiosyncratic component $\mu_{t}^{i}$,

$$
\begin{aligned}
& \hat{\mu}_{t}^{i}=\bar{\mu}_{t}+\mu_{t}^{i} \\
& \mu_{t}^{i}=\rho_{\mu} \mu_{t-1}^{i}+\epsilon_{t}^{i \mu}
\end{aligned}
$$

Finally, the process for the growth rate $\Delta \tilde{z}_{t}^{i}$ of sectoral TFP is determined by the change in a cyclical component $\Delta z_{t}^{i}$ and by a random walk stochastic trend $\gamma_{t}^{i}$

$$
\begin{aligned}
\Delta \tilde{z}_{t}^{i} & =\Delta z_{t}^{i}+\left(1+\delta_{i}\right) \gamma_{t}^{i} \\
z_{t}^{i} & =\rho_{z} z_{t-1}^{i}+\epsilon_{t}^{i z} \\
\gamma_{t}^{i} & =\gamma_{t-1}^{i}+\epsilon_{t}^{i \gamma}
\end{aligned}
$$

The auto-correlations $\rho_{\omega}, \rho_{\mu}, \rho_{z}$ belong to the interval $[0,1]$. All the shocks are assumed to be mutually independent, and their variances are indicated, respectively, with $\sigma_{\omega}^{2}, \sigma_{\mu}^{2}, \sigma_{z}^{2}$.

\subsection{Staggered prices}

In every period $t$, each monopolistically competitive firm $(i, j)$ can freely reset its price with probability $1-\theta_{i}$. Prices are indexed at the sectoral level, i.e. firms that cannot optimally reset their price mechanically increase them at an exogenous and sector specific rate $\bar{\pi}_{t}^{i}$ given $\mathrm{by}^{3}$

$$
\bar{\pi}_{t}^{i}=\bar{\pi}_{t}-\gamma_{t}^{i}
$$

\footnotetext{
${ }^{3}$ In alternative, we could make assumptions on the steady state values of aggregate factors and then obtain (10) as an equilibrium condition. For instance, without loss of generality, consider an additional factor $\psi_{i K_{m}+1} \phi_{K_{m}+1 t}=\bar{\nu}^{-1}\left(1+\delta_{i}\right) Y_{t} \mathrm{t}$ be subtracted from (6). take Take the first difference from both sides of the resulting equation and assume a steady state where real marginal costs are constant. We obtain

$$
\begin{aligned}
0 & =\sum_{k=1}^{K_{m}+1} l_{k i} \Delta \phi_{k}+\bar{\nu}^{-1}\left(1+\delta_{i}\right)[\Delta y(i)-\Delta Y]-\left(1+\delta_{i}\right) \gamma^{i} \\
& =\sum_{k=1}^{K_{m}+1} l_{k i} \Delta \phi_{k}+\left(1+\delta_{i}\right)\left[\pi-\pi^{i}-\gamma^{i}\right]
\end{aligned}
$$

where time indexes have been omitted to emphasize that the quantities are constant at the steady state. Hence if $\bar{\pi}$ is interpreted as a steady state measure of aggregate inflation $\pi$, and $\bar{\pi}^{i}$ as a steady state measure of sectoral inflation, then (10) turns out to be an equilibrium condition if we assume that $\sum_{k=1}^{K_{m}+1} l_{k i} \Delta \phi_{k}=0$. In the Example of Section 2.1, this would be equivalent to positing the following natural equilibrium condition,

$$
\sum_{k=1}^{K_{m}+1} l_{k i} \Delta \phi_{k}=\Delta(W-P-Z)-\delta_{i} \cdot \Delta(Y-N-Z)=0
$$
}


where $\bar{\pi}_{t}$ is a stochastic process defining an aggregate variable used by agents for price indexation. ${ }^{4}$ More precisely, the aggregate indexation rate $\bar{\pi}_{t}$ can be seen as stemming from purely monetary phenomena, such as the time-varying inflation target in Cogley and Sbordone (2008), or the lag of aggregate inflation as in Yun (1996). The overall sectoral indexation $\bar{\pi}_{t}^{i}$ contains, in addition, a correction for the extra long-run deflation induced in sector $i$ by its extra rate of long-run technical progress $\gamma_{t}^{i}$.

The assumptions made so far ensures that a set of sectoral NKPCs (1) is obtained using standard log-linearization techniques ${ }^{5}$. In particular, the slope $\lambda_{i}$ of the sectoral Phillips curve is

$$
\lambda_{i} \equiv \frac{\left(1-\theta_{i}\right)\left(1-\beta \theta_{i}\right)}{\theta_{i}}
$$

\subsection{Estimation challenges}

To build some intuition about the challenges in estimating the NKPC we write, after some manipulations of (1),

$$
\pi_{t}^{i}-\beta \pi_{t+1}^{i}=\lambda_{i} m_{t}^{r i}+\underbrace{\lambda_{i} \bar{\mu}_{t}+\beta e_{t+1}+\bar{e}_{t}}_{\text {Aggregate error }}+\underbrace{\lambda_{i} \mu_{t}^{i}+\beta e_{t+1}^{i}-(1-\beta) \gamma_{t}^{i}}_{\text {Idiosyncratic error }}
$$

The quantity $E_{t}\left[\pi_{t+1}^{i}\right]-\pi_{t+1}^{i}=e_{t+1}+e_{t+1}^{i}$ is the inflation forecasting error, where $e_{t+1}$ is the error part that is correlated across sectors and $e_{t+1}^{i}$ is instead i.i.d. ${ }^{6}$ Because of the assumption of rational expectation, inflation forecast errors are uncorrelated with time $t$ variables. A similar separation between aggregate an idiosyncratic components holds for the mark-up shock $\hat{\mu}_{t}^{i}$. The remaining element of (12) is the expected discounted change in the inflation indexation variable, which by virtue of (10) is split into an aggregate part $\bar{e}_{t}=$ $\bar{\pi}_{t}-\beta E_{t}\left[\bar{\pi}_{t+1}\right]$ and an idiosyncratic one $(1-\beta) \gamma_{t}^{i}$.

An inspection of (12) immediately reveals two main estimation issues. First, all the components of the unobserved error that are dated $t$ are correlated with the regressor $m_{t}^{r i}$. The LI literature has tried to solve this problem by estimating the above equation using IV-GMM methods, where lags of the observed variables are used as instruments. However, a pitfall of this strategy is that, in order for the lags to be valid instruments, mark-up shocks need to be assumed uncorrelated across time. A second issue is that the error term is likely nonstationary (Cogley and Sbordone, 2008). If, for instance, shifts in the monetary

\footnotetext{
${ }^{4}$ We will remain mostly agnostic on the properties relating the evolution of $\bar{\pi}_{t}$ to the underlying shocks to the economy. We only require $\bar{\pi}_{t}$ to be a reasonable indexation variable in the sense that, in the absence of shocks, $\bar{\pi}_{t}$ eventually converges to actual (steady state) inflation $\pi_{t}=P_{t}-P_{t-1}$. Variables that satisfy this requirement include popular cases like indexation to lagged inflation $\bar{\pi}_{t}=\pi_{t-1}$ as in Yun (1996), or to a fluctuating inflation target $\bar{\pi}_{t}=\pi_{t}^{\text {target }}$ in Cogley and Sbordone (2008).

${ }^{5}$ See Appendix A for details.

${ }^{6}$ Section 3.1 is devoted to show under what conditions a linear separation between aggregate an idiosyncratic components is warranted.
} 
policy regime are modeled as a random walk process for $\bar{\pi}_{t}$, then $\bar{e}_{t}=(1-\beta) \bar{\pi}_{t}$ is non-stationary (in addition to being correlated with $m_{t}^{r i}$ ). Estimating the NKPC in first difference might solve the non-stationarity problem. However, this would also worsen then endogeneity issue, since the first difference of the inflation forecast error $e_{t+1}+e_{t+1}^{i}$ would be correlated with $m_{t}^{r i}$.

\section{A Full Information Partial Equilibrium ap- proach}

From a theoretical perspective, the FIPE approach is developed in two steps. The first, presented in Section 3.1, provides conditions under which the marginal cost and inflation processes can be linearly separated into aggregate and idiosyncratic components. The second, outlined in Section 3.2, demonstrates that closed-form representations for the second moments of the inflation and the real marginal cost variables can be derived from the idiosyncratic components.

\subsection{Separating aggregate and idiosyncratic components}

The central aspect of our analysis is the characterization of the stochastic process for the real marginal cost $m_{t}^{r i}$ and its associated real marginal cost gap $\hat{m}_{t}^{r i}$, defined as

$$
\hat{m}_{t}^{r i}=m_{t}^{r i}+\hat{\mu}_{t}^{i}
$$

An important features of the stochastic process for $\hat{m}_{t}^{r i}$ is its persistence in response to shocks, which can be characterized by an intrinsic and an extrinsic components. For idiosyncratic shocks, the extrinsic component is equivalent to the exogenous persistences $\rho_{\omega}, \rho_{z}$ and $\rho_{\mu}$. The intrinsic component, instead, is generated purely by the presence of nominal $\left(\lambda_{i}\right)$ and real $\left(\delta_{i}\right)$ rigidities. For the rest of our analysis we divide the sectors into groups for which the following homogeneity assumption in the way nominal and real rigidities interact holds,

Assumption 1. Consider a group of sectors for which the constants $\lambda_{i}$ and $\delta_{i}$ satisfy

$$
\lambda_{i}\left(1+\delta_{i}\right)=\kappa>0
$$

Assumption 1 is slightly milder than the requirement that $\lambda_{i}$ and $\delta_{i}$ are the same across given group of sectors. For a group of sectors that share the same constant $\kappa$, the intrinsic persistence $r$ is defined as follows,

Definition 1. For a group of sectors that satisfy Assumption 1, the intrinsic persistence $r \in(0,1)$ is given by the the smaller of the two characteristic roots of second order difference equation,

$$
\hat{m}_{t+1}^{r i}-\chi_{1} \hat{m}_{t}^{r i}+\chi_{0} \hat{m}_{t-1}^{r i}=0
$$

with $\chi_{0} \equiv \beta^{-1}$ and $\chi_{1}^{i} \equiv 1+\chi_{0}(1+\kappa)$. 
Assumption 1 guarantees that the intrinsic persistence $r$ is the same across all sectors belonging to a common group. While Appendix B provides a detailed characterization of the intrinsic and extrinsic persistences, we briefly present here a more intuitive discussion.

Equation (14) is the homogeneous part of the difference equation describing the evolution of marginal cost gaps in response to aggregate and idiosyncratic shocks. The equation thus provides information about dynamic persistence $r$ of the gap's stochastic process due only to the intrinsic, endogenous propagation of the shocks caused by the nominal and real rigidities. The extrinsic persistence, instead, is derived from the inhomogeneous part of the equation, whose process depends solely on the exogenous persistence of the underlying shocks. Note that the intrinsic persistence $r$ is the same in response to both aggregate and idiosyncratic shocks. ${ }^{7}$

While for idiosyncratic shocks the extrinsic component equals the exogenous persistence $\rho_{s}$, for aggregate shocks such characterization doesn't hold anymore. This can be easily seen in our Example in Section 2.1. An aggregate structural shock, like an increase in productivity $Z_{t}$, will in fact cause a cascade of additional general equilibrium "reduced-form" shocks to other factors, like the wage $W_{t}$. It follows that the overall extrinsic persistences generated by a structural shock to $Z_{t}$ does not depend on the exogenous persistence of $Z_{t}$ alone, but also on the endogenous persistence of the response of all the other factors. Our partial equilibrium approach aims exactly at side-stepping the task of modelling explicitly such complex endogenous propagation. Note also that since the root $r$ lies within the unit circle, real marginal cost gaps, and hence sectoral inflation, could be subject to non-structural (sunspot) dynamics. This paper abstracts from the role of sunspot shocks, which could nonetheless be easily introduced into the model.

Define $\iota_{s}$ an indicator which equals -1 if $s=z$ and 1 otherwise. We then have the following result,

Proposition 1. Assumption 1 is necessary and sufficient for the real marginal cost gap $\hat{m}_{t}^{r i}$ to be linearly decomposed into aggregate and idiosyncratic components $\hat{m}_{t}^{r i}=\hat{x}_{t}^{i}+\hat{m}_{t}^{i}$ given by, respectively,

$$
\begin{aligned}
\hat{x}_{t}^{i} & =\sum_{k=1}^{K_{m}} \hat{\psi}_{k i} \hat{f}_{k t} \\
\hat{m}_{t}^{i} & =\sum_{j=0}^{\infty} \sum_{s \in\{\omega, z, \mu\}} \mathrm{a}_{j}^{s} \epsilon_{t-j}^{i s} \iota_{s}
\end{aligned}
$$

\footnotetext{
${ }^{7}$ The intuition is that, regardless the type of shock hitting the sector, (14) is always the homogeneous part of the difference equation describing the evolution of the real marginal cost gap. Hence, the intrinsic persistence $r=\frac{1}{2}\left(\chi_{1}^{2}-\sqrt{\chi_{1}^{2}-4 \chi_{0}}\right)$ is the same for all shocks. The specific type of shocks affect only the inhomogeneous part of the difference equation, which in turn determines the extrinsic persistence.
} 
where $\mathrm{a}_{j}^{s} \in(0,1)$ are functions only of $\rho_{s}$ and $r$, while $\left\{\hat{f}_{k t}\right\}$ is a set of $K_{m}>0$ aggregate factors with sectoral loadings $\left\{\hat{\psi}_{i k}\right\}$.

Proof. See Appendix B.

According to Proposition 1, the real marginal cost gap can be decomposed into a component that linearly depends only on aggregate factors, and into an idiosyncratic part which is the sum of three $\mathrm{MA}(\infty)$ processes. The explicit functional form for the weights $a_{j}^{s}$ is provided in Appendix B. Note also that an immediate consequence of Proposition 1 is that also the real marginal cost $m_{t}^{r i}$ can be decomposed into a linear combination $x_{t}^{i}$ of aggregate factors, plus an idiosyncratic part $m_{t}^{i}$ so that

$$
m_{t}^{r i}=x_{t}^{i}+m_{t}^{i}
$$

The representation (16) makes clear one important distinction between idiosyncratic demand and supply shocks on one side, and idiosyncratic mark-up shocks on the other. Consider a positive unit impulse $\epsilon_{t}^{i s} \iota_{s}$ for a shock of type $s \in\{\omega, z\}$. By (16), the shock raises the present and future values of $\hat{m}_{t}^{i}$. Therefore, by virtue of (1), the shock increases current inflation $\pi_{t}^{i}$. In particular, the shock increases both $\hat{m}_{t}^{i}$ and $m_{t}^{i}$ by an amount $\mathrm{a}_{0}^{s} \in(0,1)$. Take instead a positive unit impulse $\epsilon_{t}^{i \mu}$ to the mark-up. Again, the shock increases current inflation. However, while $\hat{m}_{t}^{i}$ is raised by $a_{0}^{\omega}$, the current real marginal cost $m_{t}^{i}=\hat{m}_{t}^{i}-\mu_{t}$ decreases by $1-\mathrm{a}_{0}^{\mu}$. In conclusion, for the purpose of characterizing the stochastic process of inflation and real marginal costs, demand and supply shocks are extremely similar, since they both cause inflation and real marginal costs to move in the same direction. Indeed, if we take $\rho_{\omega}=\rho_{z}$, then $\mathrm{a}_{j}^{\omega}=\mathrm{a}_{j}^{z}$ and the effects on $\pi_{t}^{i}$ and $m_{t}^{i}$ of two shocks are completely indistinguishable. Mark-up shocks, instead, introduce distinctive dynamics by causing inflation and real marginal costs to move in opposite directions. This is a standard result for New-Keynesian models, where only mark-up shocks generate a policy trade-off between output and inflation stabilization (Clarida et al. 1999). We will return to this point in the next section.

Similarly to what we have done with the marginal cost gap, define the inflation gap $\tilde{\xi}_{t}^{i}$ as

$$
\tilde{\xi}_{t}^{i}=\pi_{t}^{i}-\beta \pi_{t+1}^{i}
$$

We then have the following Corollary to Proposition 1

Corollary 1. The process for the inflation gap $\tilde{\xi}_{t}^{i}$ can be decomposed into an aggregate and an idiosyncratic component $\tilde{\xi}_{t}^{i}=\tilde{x}_{t}^{i}+\xi_{t}^{i}$ given by,

$$
\tilde{x}_{t}^{i}=\sum_{k=1}^{K_{\xi}} \tilde{\psi}_{k i} \tilde{f}_{k t}
$$


where $\tilde{f}_{k t}$ for $k=1, \ldots, K_{\xi}$ indicate a set of aggregate factors and $\tilde{\psi}_{k i}$ are a set of weights. The idiosyncratic component $\xi_{t}^{i}$ satisfies the system of equations

$$
\begin{aligned}
\xi_{t}^{i} & =\lambda_{i} m_{t}^{i}+u_{t}^{i} \\
u_{t}^{i} & =\lambda_{i} \mu_{t}^{i}+\beta e_{t+1}^{i}-(1-\beta) \gamma_{t}^{i} \\
e_{t+1}^{i} & =\epsilon_{t+1}^{i \gamma}-\lambda_{i} \sum_{s \in\{\omega, z, \mu\}} \frac{1-\mathrm{a}_{0}^{s}}{\kappa} \epsilon_{t+1}^{i s} \iota_{s}
\end{aligned}
$$

Proof. See Appendix B.

In empirical applications of our FIPE methodology, Proposition 1 and Corollary 1 are used as a preliminary step to separate aggregate and idiosyncratic components of the real marginal cost and inflation gaps data. In practice, this separation requires the use of econometric techniques, such as principal components methods. Assumption 1, by guaranteeing the validity of Corollary 1, provides necessary and sufficient conditions that justify the FAVAR model that Boivin et al. (2009) employ to study the inflation process at the sectoral level.

The results of this section can also be cast in the language of the panel literature. Every group of sectors to which Assumption 1 applies form a panel data. Corollary 1 implies that the dependent variable $\pi_{t}^{i}-\beta \pi_{t+1}^{i}$ in the linear model (12) has the multifactor error structure studied in Pesaran (2006). However, the estimation of (12) with the panel methodology in Pesaran (2006) is problematic since, as pointed out in Section 2.3, in our case the idiosyncratic error terms are correlated with the (idiosyncratic) sectoral regressor $m_{t}^{r i}$. The next section presents FIPE moments that exploit the model's structural equations to take such correlation into account.

\section{$3.2 \quad$ FIPE moments}

In this section we establish the existence of closed form expressions for the projection of the inflation gaps $\xi_{t}^{i}$ on any lag (or lead) $j$ of the real marginal cost $m_{t-j}^{i}$, with $j$ an integer. We show that the projections are linear in the sectoral slope $\lambda_{i}$ and non-linear in the intrinsic persistence $r$ and in the relative variance $R$, to be defined below, of the idiosyncratic mark-up shocks. Projection coefficients are at the heart of many econometric methods, so our closed-form representations for the projection coefficients can be applied in a variety of estimation contexts (GMM and MLE are, for instance, natural candidates).

Corollary 2. Take $\rho_{\omega}=\rho_{z} \equiv \rho$. Then, for a given value of $\rho$, the projections of $\xi_{t}$ onto $m_{t-j}^{i}$, for $j$ any integer number, can be expressed as

$$
\frac{\operatorname{Cov}\left(\xi_{t}^{i}, m_{t-j}^{i}\right)}{\operatorname{Var}\left(m_{t-j}^{i}\right)}=\lambda_{i} b_{j}(r, R)
$$


where each function $b_{j}(r, R)$ has a closed form expression and $R$ is the relative variance of the mark-up shock, i.e.

$$
R \equiv \frac{\sigma_{\mu}^{2}}{\sigma_{\omega}^{2}+\sigma_{z}^{2}+\sigma_{\mu}^{2}}
$$

The representation (20) holds even when the projections are performed with variables in differences.

Proof. See Appendix B, which also provides the closed form expression for the functions $b_{j}(r, R)$ at any (positive or negative) lag $j$.

Corollary 2 is based on the assumption that $\rho_{\omega}=\rho_{z}$, i.e. that exogenous demand and supply shocks have the same auto-correlation. In our context this is a mild assumption since, as noted in the discussion of Proposition 1, demand and supply shocks already play an almost identical role in defining the stochastic process for $m_{t}^{i}$ and $\xi_{t}^{i}$. The assumption $\rho_{\omega}=\rho_{z}$ exploits to the full extent the symmetric role of the two shocks by guaranteeing that $\mathrm{a}_{j}^{\omega}=\mathrm{a}_{j}^{z}$. In this way, the stochastic processes for $\xi_{t}^{i}$ and $m_{t}^{i}$ depend only on $\epsilon_{t}^{i \omega}-\epsilon_{t}^{i z}$ and on $\epsilon_{t}^{i \mu}$ separately. What matters for the projections is then just the relative variance $R$ of the mark-up shock.

Note also that the formulation (20) holds even when the moments are calculated using lagged variables. This is a convenient result in light of the observation that the idiosyncratic error $u_{t}^{i}$ in (19) is in general non-stationary, an issue that can be tackled by estimating the NKPCs in first differences. In this case, the first differences $\Delta e_{t+1}^{i}$ of the forecast errors become an additional variable endogenous to the regressor $\Delta m_{t}^{i}$. This is not a problem, however, since the FIPE moments built according to Corollary 2 take into account this additional form of endogeneity of the error terms.

Finally, Corollary 2 allows us also to obtain explicit moments involving the auto-covariance of $\xi_{t}^{i}$. These conditions turn out to be especially useful when (19) is expressed in first differences $\Delta \xi_{t}^{i}$. The differencing operator, in fact, introduces auto-correlation in the shocks $\Delta u_{t}^{i}$. In this instance, the auto-covariances become a function also of the relative variance $\Gamma$ of the trend shocks $\epsilon_{t+1}^{i \gamma}$, which for our purposes can be treated as pure measurement error. ${ }^{8}$ For instance, define

$$
\Gamma=\frac{\operatorname{Var}\left(\Delta \tilde{\epsilon}_{t}^{i \gamma}\right)}{\operatorname{Var}\left(\Delta e_{t}^{i}\right)}
$$

so that $\Gamma \in[0,1)$ is the contribution of the (normalized) variable $\Delta \tilde{\epsilon}_{t}^{i \gamma} \equiv \Delta \epsilon_{t}^{i \gamma} / \lambda_{i}$ to the overall volatility of the forecast error $\Delta e_{t}^{i}$. We can then write the following

\footnotetext{
${ }^{8}$ To make this interpretation more straightforward, assume that $(1-\beta) \Delta \gamma_{t}^{i}=(1-\beta) \epsilon_{t}^{i} \approx 0$. This approximation is reasonable since, in our calibration, $\beta \approx 1$ and moreover, under our structural interpretation, the shocks $\epsilon_{t}^{i}$ should be relatively small. With this approximation in mind, notice that, within the error term $\Delta u_{t}^{i}$, the variable $\Delta \epsilon_{t}^{i \gamma}$ is independent from $\Delta m_{t}^{i}$. We can then interpret the shocks $\epsilon_{t+1}^{i \gamma}$ in $u_{t}^{i}$ as picking up both trend growth shocks and true (possibly large) measurement error.
} 
additional moment conditions,

$$
\frac{\operatorname{Cov}\left(\Delta \xi_{t}^{i}, \Delta \xi_{t-j}^{i}\right)}{\operatorname{Var}\left(\Delta m_{t}^{i}\right)}=\lambda_{i}^{2} \hat{b}_{j}(r, R, \Gamma)
$$

for any integer $j$ and for given functions $\hat{b}_{j}(r, R, \Gamma){ }^{9}$

\subsection{Beyond the pure forward-looking model}

In search for formulations that can better fit the data, the literature has departed from the pure forward-looking NK model and has proposed alternative versions of the Phillips curve and of the real marginal cost. A strength of the FIPE methodology is that it can be flexibly adapted to these cases.

For instance, while (1) already incorporates in $\bar{\pi}_{t}$ popular types of backwardlooking inflation indexation, the framework can be extended to allow for indexation to past sectoral inflation $\pi_{t-1}^{i}$. This can be accomplished by adding a term $\pi_{t-1}^{i}$ to the right side side of (1), giving rise to a hybrid model (Galí and Gertler, 1999). Similarly, the real marginal cost function (6) could be made dependent also on lagged values $y_{t-1}(i)$ of sectoral output to account for a delayed effect of sectoral wage bargaining or for other sectoral adjustment costs. For instance, Batini et al. (2005) show that the introduction of labor adjustment costs can improve the empirical performance of the Phillips curve.

For both these alternative formulations, the FIPE approach would be implemented as follows. First, the introduction of lagged variables implies that (14) is now of third order. Hence, Definition 1 might have to be modified to take into account the potential presence of multiple roots (i.e. multiple intrinsic persistences $r$ ) that lie within the unit circle. Correspondingly, Assumption 1 would also have to be adjusted to ensure that all such roots are the same within sector groups. With these modifications, the moving average decomposition in Proposition 1 is still valid. Of course, the weights $\mathrm{a}_{j}^{s}$ in Proposition 1 may now depend on the multiple intrinsic persistences $r$. If this is the case, then the new moment conditions would also be a function of such multiple roots.

\section{FIPE in a Bayesian application}

In this section we provide an empirical application of the theoretical results of the FIPE approach developed above. Specifically, the results of Section 3.1 are first used to remove aggregate shock trough standard principal components methods. In turn, the moments representation of Section 3.2 is exploited within a Bayesian estimation context. Our application is based on calibrations for the extrinsic persistences $\rho_{s}$ that lead to an $\operatorname{ARMA}(1,1)$ process for the real marginal cost gaps.

\footnotetext{
${ }^{9}$ Appendix C explicitly derives this formulation for the empirical analysis in Section 4.
} 


\subsection{An ARMA(1,1) specification}

A Bayesian approach to the use of the FIPE moments is particularly attractive because the projections $b(r, R)$ depend on two parameters - the intrinsic persistence $r$ and the relative variance $R$ - which have intuitive interpretations and for which some prior knowledge can be elicited. With the aim of providing an even sharper intuition for the parameters $(r, R)$ we make the assumption that all shocks have the same persistence, and follow either a random walk or of an i.i.d. process,

$$
\begin{aligned}
\rho_{\omega} & =\rho_{z}=\rho_{\mu} \equiv \rho \\
\rho & \in\{0,1\}
\end{aligned}
$$

The assumptions above may appear restrictive, but they still allow us to move beyond the i.i.d. case typically analyzed in the LI literature. In particular, estimating the model under the two polar possibilities $\rho \in\{0,1\}$ can shed light on the sensitivity of the estimated NKPC's slopes to different assumptions about the persistence of the underlying shocks. Moreover, the above calibration for the extrinsic persistences lead to a stochastic process for the forcing variable $\hat{m}_{t}^{i}$ that is indeed quite general, i.e.

$$
\begin{aligned}
\hat{m}_{t}^{i} & =r \hat{m}_{t-1}^{i}+\epsilon_{t}-\frac{r(1-\rho)}{\mathrm{a}_{0}} \epsilon_{t-1} \\
\epsilon_{t} & \equiv \mathrm{a}_{0}\left(\epsilon_{t}^{i \omega}-\epsilon_{t}^{i z}+\epsilon_{t}^{i \mu}\right)
\end{aligned}
$$

The real marginal cost gap then follows an $\operatorname{ARMA}(1,1)$. The intrinsic persistence $r$ is identified as the coefficient of the $\mathrm{AR}(1)$ part, while the MA(1) part disappears in the random walk case $\rho=1$. The coefficient $\mathrm{a}_{0}^{s}$ now takes the same value for all types of shocks and is thus indicated simply with $\mathrm{a}_{0}$. Moreover, the coefficient $R$ for the relative variance takes an even more intuitive meaning: it is the share of variance of the forcing variable $\hat{m}_{t}^{i}$ attributable to mark-up shocks. We can then use the expression (22) to elicit prior distributions for $R$ and $r$ in the following way.

First, the New Keynesian literature usually assigns a non-negligible role to mark-up shocks in explaining the variability of the aggregate forcing variable. Our prior is that this intuition carries over to the idiosyncratic component $\hat{m}_{t}^{i}$. We then construct a loose prior distribution for $R$ around a modal value of $1 / 3$, where mark-up shocks are as important as the other two shocks in determining the variability of $\hat{m}_{t}^{i}$.

Second, recall that, in the discussion about Assumption 1, it was pointed out that the intrinsic persistence $r$ is the same in response to both aggregate and idiosyncratic shocks. Our prior for $r$ is then constructed as a distribution centered around the estimated $\operatorname{AR}(1)$ coefficient of an $\operatorname{ARMA}(1,1)$ model, fitted to the time series of an aggregate proxy for $\hat{m}_{t}^{r i} \cdot{ }^{10}$ Specifically, as often done

\footnotetext{
${ }^{10}$ There is clearly no reason to believe that the aggregate real marginal cost gap follows exactly the process (22). In fact, even if we assume that aggregate structural shocks follow
} 
in the literature, the proxy is taken to be a measure of the output gap. ${ }^{11}$ More details about the prior distributions, including the prior for $\lambda_{i}$, are provided in the next section.

The likelihood of the data is obtained as follows. First of all, to address the problem of non-stationarity, we estimate (19) in first differences, namely we use projections of $\Delta \xi_{t}^{i}$ on leads and lags of $\Delta m_{t}^{i}$ and of $\Delta \xi_{t}^{i}$ itself. Specifically, we use a number $2 j+1$ of symmetric leads and lags of $\Delta m_{t}^{i}$, and a number $J$ of lags of $\Delta \xi_{t}^{i}$. We collect these variables into a column vector $X_{t}^{i}$,

$$
X_{t}^{i}=\left[\begin{array}{llllllll}
\Delta \xi_{t-J}^{i} & \ldots & \Delta \xi_{t-1}^{i} & \Delta m_{t-j}^{i} & \ldots & \Delta m_{t}^{i} & \ldots & \Delta m_{t+j}^{i}
\end{array}\right]^{\prime}
$$

Define $\Sigma_{a}$ the covariance matrix of the elements of $X_{t}^{i}$, and $\Sigma_{b}$ the row vector collecting the projections (20)-(21) scaled by $\lambda_{i}$,

$\Sigma_{b}=\left[\begin{array}{llllllll}\lambda_{i} \hat{b}_{J}(r, R, \Gamma) & \ldots & \lambda_{i} \hat{b}_{1}(r, R, \Gamma) & b_{j}(r, \hat{R}) & \ldots & b_{0}(r, \hat{R}) & \ldots & b_{-j}(r, \hat{R})\end{array}\right]$

The analytic expressions for $\Sigma_{a}$ and $\Sigma_{b}$ are provided in Appendix C. Define $\mathrm{b}\left(\lambda_{i}, r, R, \Gamma\right)$ the vector of regression coefficients of $\Delta \xi_{t}^{i}$ on $X_{t}^{i}$,

$$
\mathrm{b}\left(\lambda_{i}, r, R, \Gamma\right)=\Sigma_{b} \Sigma_{a}^{-1}
$$

and call $\bar{\sigma}_{i}^{2}$ the variance of the residuals of such regression. We estimate $\bar{\sigma}_{i}^{2}$ directly from the data.

Assume that the i.i.d. shocks $\epsilon^{\omega}, \epsilon^{z}, \epsilon^{\mu}$ and $\epsilon^{\gamma}$ are jointly normally distributed. Proposition 1 then implies that idiosyncratic real marginal costs are jointly normally distributed with respect to each other and with respect to the inflation gaps $\xi_{t}$. It follows that,

$$
\Delta \xi_{t}^{i} \mid X_{t}, \lambda_{i}, r, R \sim N\left(\lambda_{i} \mathrm{~b}\left(\lambda_{i}, r, R, \Gamma\right) X_{t}^{i}, \bar{\sigma}_{i}^{2}\right)
$$

Suppose that we have a sample of $T$ observations for each sector $i$. We approximate the sectoral likelihood $L_{i}(\cdot \cdot \cdot)$ of the data as

$$
L_{i}\left(\Delta \xi_{0}^{i}, \ldots, \Delta \xi_{T}^{i} \mid \Delta m_{0}^{i}, \ldots, \Delta m_{T}^{i}, \lambda_{i}, r, R, \Gamma\right) \approx \prod_{t=0}^{T} \mathrm{f}_{i}\left(\Delta \xi_{t}^{i} \mid X_{t}^{i}, \lambda_{i}, r, R, \Gamma\right)
$$

where $f_{i}(\cdot \mid \cdot)$ is the probability density function of the normal distribution (24). The likelihood contains an approximation, since each $f_{i}(\cdot \cdot \cdot)$ is conditioned on

either an i.i.d. or a random walk processes, still the aggregate factors would not in general be i.i.d. or random walk. As discussed in Section 3.1, this is do to the presence of general equilibrium effects, which induce endogenous persistence in the factors beyond that imposed on the exogenous structural shocks. This conclusion notwithstanding, we consider the procedure good enough to be used with the limited goal of eliciting a central value for the prior distribution of $r$.

${ }^{11}$ Note that we are not estimating the slope of the Phillips curve by projecting inflation on measures of the output gap. This can be problematic as pointed out, for instance, by Galí and Gertler (1999). Instead, we are just using the often exploited theoretical relation between marginal cost gaps and output gaps to merely infer something about the auto-correlation of the former. Finally, in our exercise an $\operatorname{ARMA}(1,1)$ fits quite well our output gap series. 
$X_{t}^{i}$, which contains only a subset of the history, up to time $t$, of first differences of real marginal costs and of inflation gaps. This issue can be accommodated by choosing lags $j$ and $J$ large enough.

To increase the number of observations used in the estimation, we employ a pooled version of the sectoral likelihood. Assume that the slopes $\lambda_{i}$ are drawn from a distribution with mean $\bar{\lambda}$. The draws are taken to be independent of all the idiosyncratic sectoral shocks. Then, by pooling sectors together, we can define a new likelihood as,

$$
L(\boldsymbol{\Delta} \boldsymbol{\xi} \mid \boldsymbol{\Delta} \boldsymbol{m}, \bar{\lambda}, r, R, \Gamma)=\prod_{i} L_{i}\left(\Delta \xi_{0}^{i}, \ldots, \Delta \xi_{T}^{i} \mid \Delta m_{0}^{i}, \ldots, \Delta m_{T}^{i}, \bar{\lambda}, r, R, \Gamma\right)
$$

where $\Delta \boldsymbol{\xi}$ and $\Delta \boldsymbol{m}$ represent the entire collection of time and sector observations of first differences of inflation gaps and real marginal costs. Note that the multiplication in $L(\cdot \mid \cdot)$ of sectoral likelihoods is justified since, at this stage, all aggregate shocks have been removed, and hence the evolution of the observables are independent across sectors.

For given values of $\bar{\sigma}_{i}^{2}$, the posterior density $\mathrm{p}(\bar{\lambda}, r, R, \Gamma)$ of the parameters is given by,

$$
\mathrm{p}(\bar{\lambda}, r, R, \Gamma)=L(\boldsymbol{\Delta} \boldsymbol{\xi} \mid \boldsymbol{\Delta} \boldsymbol{m}, \bar{\lambda}, r, R, \Gamma) \mathrm{p}_{0}(\bar{\lambda}, r, R, \Gamma)
$$

where $\mathrm{p}_{0}(\bar{\lambda}, r, R, \Gamma)$ is the prior distribution of the parameters.

\subsection{Data description and priors}

The data source we employ is the EU KLEMS Database, March 2008 release. The database contains information for 3-digit industries at annual frequency for the period 1970-2005. We consider three groups of industries, corresponding to the categorization in Basu et al. (2006): Non-Durable manufacturing (sector group 1), Durable Manufacturing (sector group 2), Non-Manufacturing (sector group 3). Groups are of roughly equal size of 10 industries each. We posit that Assumption 1 holds within each group.

First differences of inflation gaps are constructed from sectoral log price of gross output, using a discount parameter calibrated to a standard annual value of $\beta=0.97$. First differences in the real marginal cost are calculated from the log labor share in gross output. Our estimation thus tests the labor share-based, purely forward looking Phillips curve in Galì and Gertler (1999) and Cogley and Sbordone (2008). For each group of sectors, common factors are extracted from the series via standard principal components procedures, with the number of components selected according to the ER test in Bai and $\mathrm{Ng}$ (2002). The remaining idiosyncratic series, used in the estimation, typically explan 20-30\% of the variance of the original series.

In the construction of the vectors $X_{t}^{i}$ we select $j=J=1$. Standard deviations $\bar{\sigma}_{i}$ are calibrated using the preliminary regression described in the previous section. To assess whether a number $J=1$ of lags is appropriate, we 
test whether the regression errors display auto-correlation and we conclude that they largely don't.

Priors are set as follows. Due to the significant uncertainty found in the empirical literature, we choose an agnostic prior for the distribution of $\bar{\lambda}$, which is assumed to be a normal centered at 0 , with a large standard deviation equal to 3. For the intrinsic persistence $r$, we select a distribution with a mode of 0.5 and a two-sided $90 \%$ interval $(.25, .65)$. The value 0.5 is obtained by running an $\operatorname{ARMA}(1,1)$ on the OECD output gap series from 1995 to 2016 for the US, Japan and UK, and averaging the corresponding AR(1) coefficients across the three countries. For $R$ we choose a distribution with mode at $1 / 3$, corresponding to the prior that mark-up shocks are as important as each each of the two other shocks in explaining variation in $\hat{m}_{t}^{i}$, with a two-sided $90 \%$ interval $(.08, .54)$. For the relative variance $\Gamma$ of the measurement error we don't have a clear prior, so we experiment with various alternatives. In general, due to the reasonably large number of observations (about 330) available for each group, and because of further considerations discussed below, the posterior distribution of $\lambda$ is quite insensitive to the priors for $r, R$ and $\Gamma$. We therefore defer to Appendix D for a more in-depth discussion of the priors and posteriors for these three parameters. ${ }^{12}$

\subsection{Estimation results}

To discuss the estimation results, we need first to identify a target value for the slope $\bar{\lambda}$, against which to assess whether our estimates indicate that the forward-looking Phillips curves are supported by the data. However, finding an appropriate target is here complicated by the fact that sectoral data are usually available only at annual frequency. Virtually all the macro literature, instead, has focused on debating target slopes at quarterly frequency.

A well-known problem with empirical estimates of the slope of the NKPC is that, even when estimates return a positive coefficient, the implied degree of price stickiness appears too large to be consistent with the micro evidence. However, the the appropriate degree of microeconomic price stickiness is also subject to considerable debate. To take all these uncertainties into consideration, we then present three measures - main, conservative, very conservative of target values for $\lambda$.

The main target measure is constructed by assuming a monthly probability of price adjustment equal to .2 , which implies a monthly $\theta_{m}=.8$. This value is consistent with the median frequency of adjustment for posted prices reported in Nakamura and Steinsson (2008). The argument for focusing on posted prices, instead of regular prices, can be defended by recalling that our identification scheme relies on responses to idiosyncratic shocks, which should be associated with much more price flexibility than aggregate shocks (Boivin et al. 2009, Kehoe and Midrigan 2015, Nakamura and Steinsson 2013, Carvalho

\footnotetext{
${ }^{12}$ In particular, the priors for $r$ and $R$ are obtained from gamma distributions of appropriate monotonic transformations $\hat{r}=r /(1-r)$ and $\hat{R}=R /(1-R)$. Similarly, the prior for $\Gamma$ is derived from a uniform distribution of a transformation $\hat{\Gamma}$.
} 
2006). Monthly frequencies are then cumulated geometrically to obtain an annual value of $\theta_{a}=.8^{12}=0.07$ (see Dennis 2006 for a discussion). In turn, these calculations yield an annual slope $\lambda_{a}$ equal to 12 . In an attempt to reconcile the low estimates for $\lambda$ with the micro evidence, the literature has also emphasized that, in the presence of real rigidities, the estimated parameter $\hat{\lambda}$ should be equal to the true value $\lambda$ times a constant. For instance, in her baseline calibration of real rigidities, Sbordone (2002) obtains $\hat{\lambda}=\frac{1}{3} \lambda$. To err on the side of caution, we apply this relation to the calculations above, to yield a main target value of $\lambda_{a}=4$.

Following the steps above, our conservative measure is computed from $\theta_{m}=$ .86 , or a monthly probability of adjustment equal to .14, which corresponds to the median frequency of adjustment for regular prices reported in Klenow and Kryvtsov (2008). The implied annual slope, adjusted for the usual constant, is $\lambda_{a}=1.5$.

Similarly, a very conservative can derived by setting $\theta_{m}=.90$, corresponding to the monthly price stickiness for regular prices in Nakamura and Steinsson (2008). ${ }^{13}$ After adjusting for the constant, we obtain an implied $\lambda_{a}=0.6$. We can also provide an additional derivation for our very conservative measure of the target slope. It is clear that the geometric transformations of the monthly Calvo parameter is subject to a large non-linearity, with the target slope rising quickly as the time horizon increases. To reduce this non-linearity, we postulate that the real marginal cost gap follows a monthly $\mathrm{AR}(1)$ process, with autoregressive parameter equal to $r_{m}=0.94$ (corresponding to the annual $r_{a}=.5$ used, in the previous section, as mode for the prior). The monthly slope is set to $\lambda_{m}=0.02$, obtained from the conservative $\theta_{m}=0.86$. Next, we ask what would be the $\lambda_{a}$ obtained by trying to fit, at an annual frequency, the original process of

\footnotetext{
${ }^{13}$ This calibration also comes close to the counterfactual average duration of 12 months calculated by Kehoe and Midrigan (2015).
} 

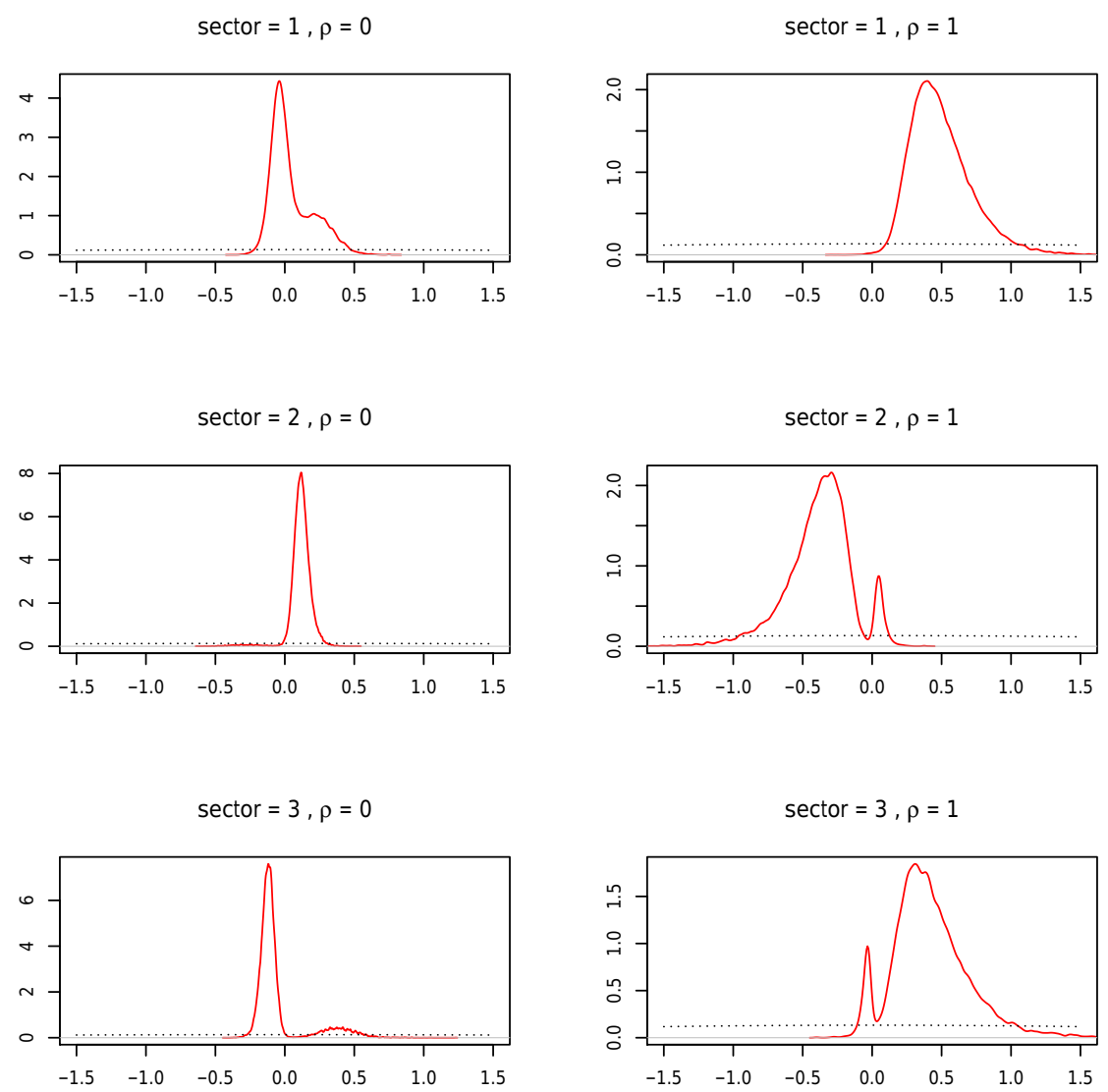

Figure 1: Smoothed posterior density of $\bar{\lambda}$ for the USA. For each of the three sector groups, estimation are carried out for the two configurations $\rho=0$ and $\rho=1$ of the extrinsic persistence. Dotted lines indicate prior densities.

the Phillips curve variables. ${ }^{14}$ The answer is a fitted annual slope equal to 2.1,

\footnotetext{
${ }^{14}$ Time is measured in months. For brevity, assume that after month $t$ the monthly real marginal cost gap $\hat{m}_{t}$ follows a deterministic AR(1) path. A standard NKPC can be written as

$$
\pi_{t}=12 \lambda_{m} \hat{m}_{t}^{a}+\beta E_{t} \pi_{t+12}
$$

where $\hat{m}_{t}^{a} \equiv \frac{1}{12} \sum_{j=0}^{11} \beta_{m}^{j} \hat{m}_{t+j}$ indicates the average annual real marginal cost gap over the year beginning in month $t$, and $\beta \equiv \beta_{m}^{12}$ is the annual discount. Since $\beta$ is very close to 1 , the discounting in $\hat{m}_{t}^{a}$ can in practice be disregarded. After summing the above expression over 12 consecutive months we finally have

$$
\pi_{t}^{a}=\left(12 \sum_{j=0}^{11} r_{m}^{j}\right) \lambda_{m} \hat{m}_{t}^{a}+\beta E_{t}^{a} \pi_{t+1}^{a}
$$
}



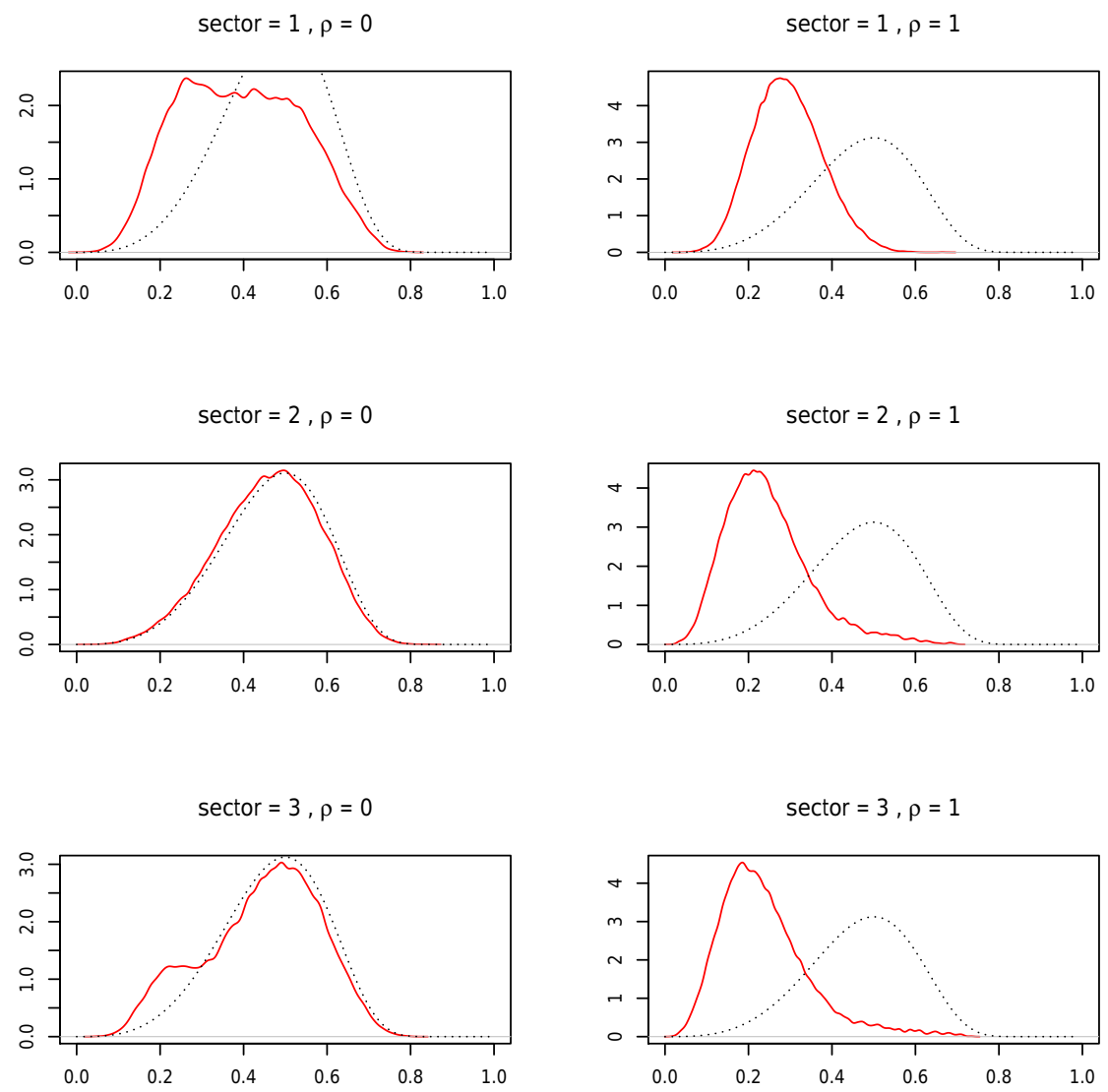

Figure 2: Smoothed posterior density of $r$ for the USA. For each of the three sector groups, estimation are carried out for the two configurations $\rho=0$ and $\rho=1$ of the extrinsic persistence. Dotted lines indicate prior densities.

which divided by the usual constant gives again a value around $\lambda_{a}=.6$.

We present estimations for two large economies, the US and Japan, and for a small open economy, the UK. Posterior distributions are approximated using a

where inflation over the year beginning in $t$ is $\pi_{t}^{a} \equiv \sum_{j=0}^{11} \pi_{t+j}$, and annual expected inflation is the sum of the one-year-ahead inflation expectations surveyed between months $t$ and $t+11$, namely $E_{t}^{a} \pi_{t+1}^{a} \equiv \sum_{j=0}^{11} E_{t+j} \pi_{t+12+j}$. Note that we have exploited the structure $\hat{m}_{t+j}^{a}=$ $r_{m}^{j} \hat{m}_{t}^{a}$ stemming from the deterministic $\operatorname{AR}(1)$ dynamics of $\hat{m}_{t}$. The fitted relation between the slopes at annual and monthly frequencies is then

$$
\lambda_{a}=\left(12 \sum_{j=0}^{11} r_{m}^{j}\right) \lambda_{m} \approx 107 \lambda_{m}
$$



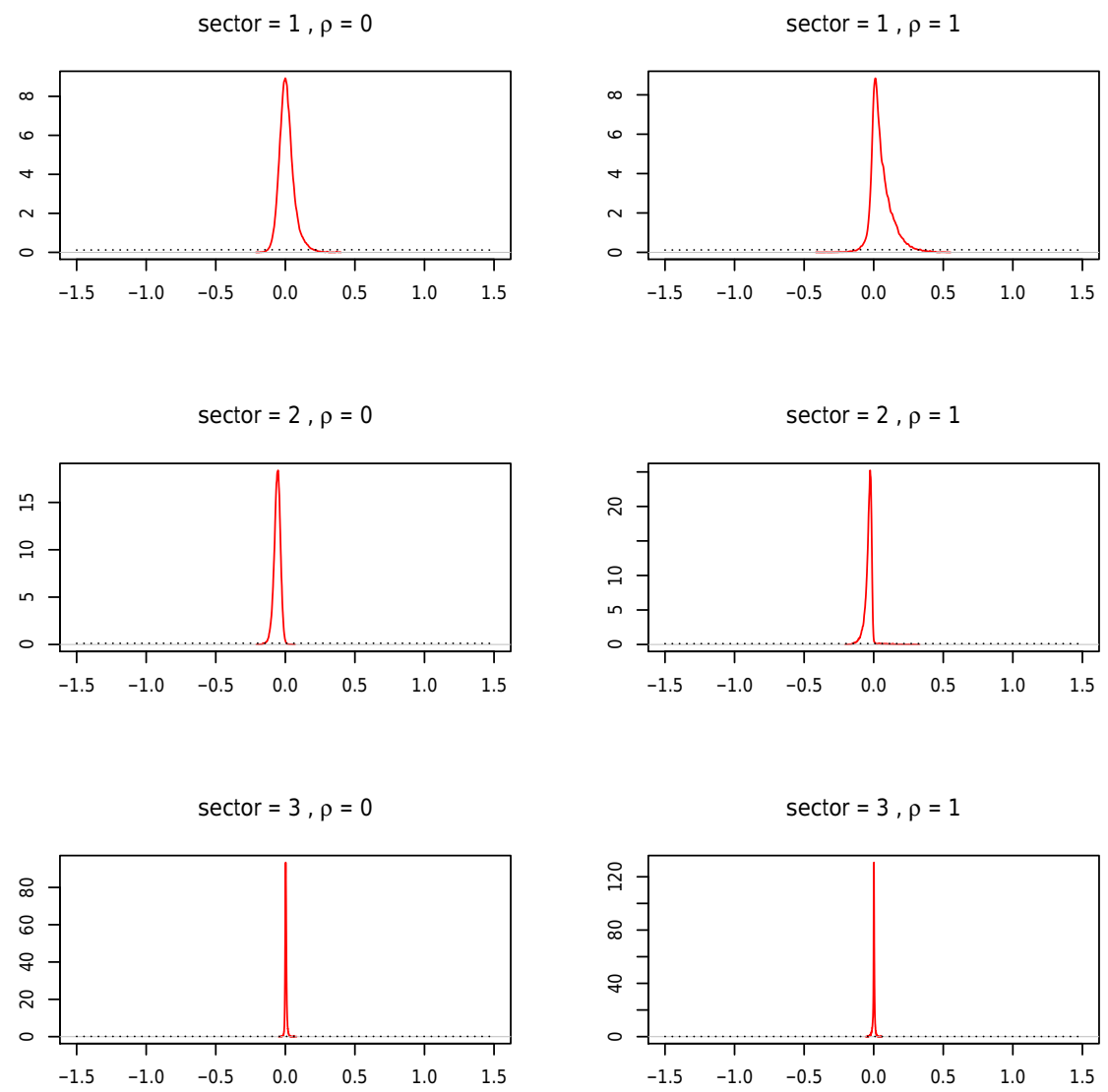

Figure 3: Smoothed posterior density of $\bar{\lambda}$ for Japan. For each of the three sector groups, estimation are carried out for the two configurations $\rho=0$ and $\rho=1$ of the extrinsic persistence. Dotted lines indicate prior densities.

Random Walk Metropolis-Hastings algorithm with 500,000 draws. ${ }^{15}$ We begin with a discussion of the results for the US, which have been the focus of much of the early empirical NK literature.

Figure 1 depicts the (smoothed) posterior density of the average slope $\bar{\lambda}$ for the three separate sector groups. Sectoral slopes are estimated under the two alternative configurations $\rho=0$ and $\rho=1$ for the extrinsic auto-correlation of the shocks. The main takeaway from the figure is that posterior distributions for $\bar{\lambda}$ are overall inconsistent with our target values. In fact, for all parameter configurations, the posterior distributions are concentrated well below our main

\footnotetext{
${ }^{15}$ We employ an adaptive algorithm for the covariance matrix of the proposal distribution. The first 10,000 draws are discarded.
} 

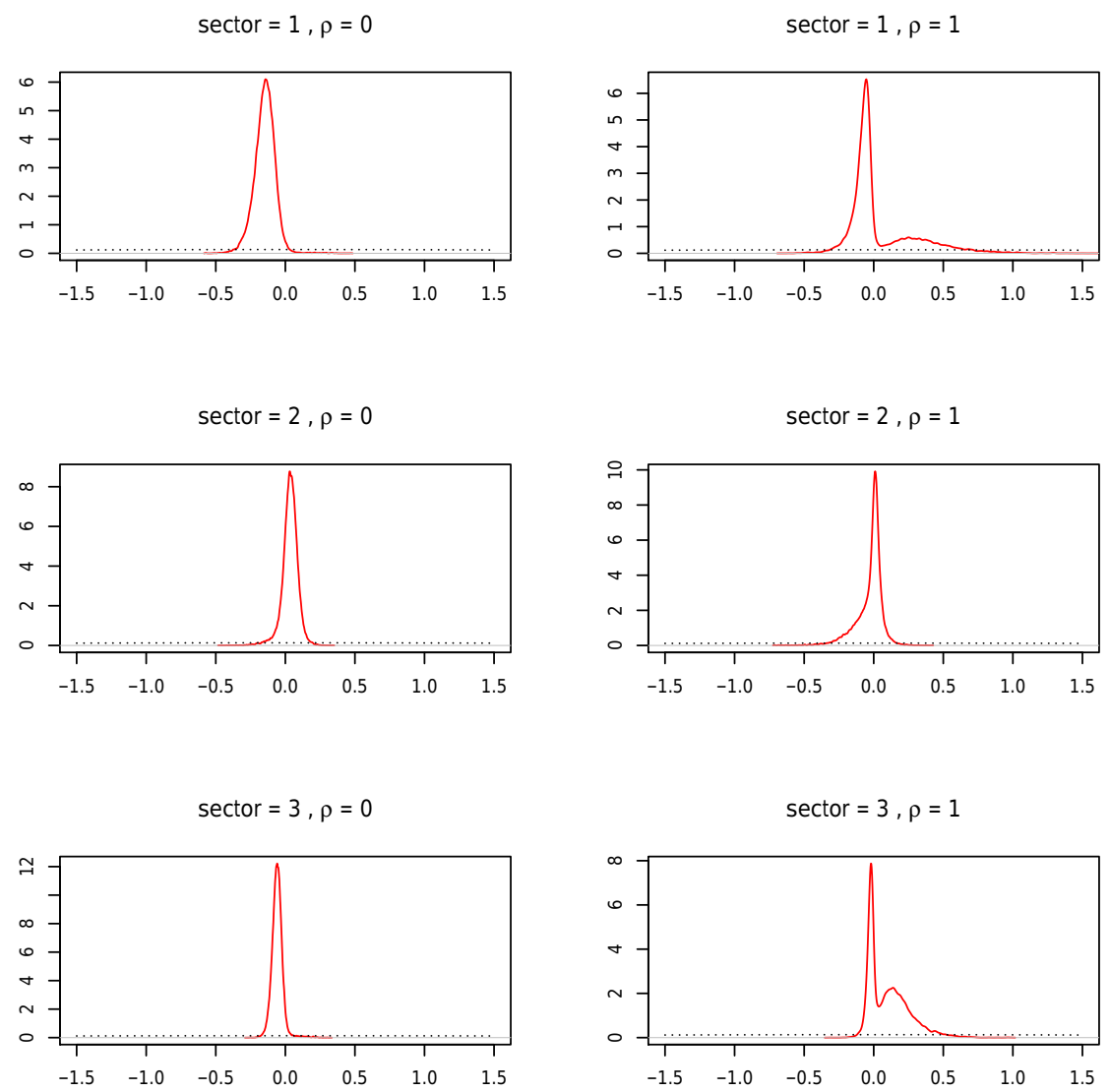

Figure 4: Smoothed posterior density of $\bar{\lambda}$ for the UK. For each of the three sector groups, estimation are carried out for the two configurations $\rho=0$ and $\rho=1$ of the extrinsic persistence. Dotted lines indicate prior densities.

(4) and conservative (1.5) targets. Only the very conservative target (0.6) falls meaningfully within the posterior density for the case $\rho=1$ and for the first and third sector groups. However, even for this configuration of parameters, the posterior of the second sector group (Durable manufacturing) is concentrated on negative values. Negative values are also indicated for the third sector group (Non-Durable manufacturing) under the assumption of i.i.d. shocks. Therefore, differently than Galí (1999) and Sbordone (2002), our estimations reject the labor share-based, purely forward-looking NKPC for the US. Our conclusions are instead in line with the literature that finds either excessive implied price stickiness, or even negative slopes for the NKPC.

Posterior densities for the remaining model parameters are reported in Ap- 
pendix D. For brevity, we present in Figure 2 only the posteriors for $r$. Notice that for the sector group 1 posterior densities track closely the respective prior. This conclusion extends also to the posteriors of $R$ and $\Gamma$. The explanation is that for, $\rho=0$, posterior densities of $\bar{\lambda}$ are concentrated on values very close to zero. It is easily seen that, for $\bar{\lambda}=0$, the projection coefficients (23) of the inflation gap onto the real marginal cost are zero, which correctly indicates that there is no relation between inflation and real marginal costs. Hence, while the moments conditions point with high precision to values of $\bar{\lambda}$ close to zero, they cannot be informative about the distribution of the other parameters.

Finally, Figures 3 and 4 report estimation results for Japan and the UK, respectively. ${ }^{16}$ For both countries, and for all values of $\rho$, the slope $\bar{\lambda}$ is very precisely estimated to be concentrated around zero in all sector groups, well below even our most conservative target measure.

\section{Conclusions}

Econometric tests of the validity of the New Keynesian Phillips Curve have yielded conflicting findings, often at odds with the microeconomics evidence. The likely reason is that estimates on aggregate data are subject to significant challenges, due to the presence of omitted variables and model misspecifications.

I propose a new approach to estimation, which exploits the extensive sectoral datasets that have become available in recent years. The new methodology aims at leveraging the advantages of both the structural Full Information and the single-equation Limited Information literatures. The identification strategy is based on modeling the partial equilibrium responses to a variety of sectoral idiosyncratic shocks. For these reasons, the methodology can be characterized as Full Information Partial Equilibrium (FIPE).

The FIPE approach is flexible along two lines. First, it is easily extended to alternative hybrid versions of the NKPC. Second, the theoretical moment conditions delivered by the FIPE analysis can be employed as an input to a variety of econometric techniques.

Since the FIPE identification is based on idiosyncratic shocks, a preliminary step to estimation requires the purging of sectoral time series of the effects of aggregate shocks. To this end, I provide necessary and sufficient conditions on the assumed interaction between nominal and real rigidities that allow inflation and marginal cost time series to be linearly separated into aggregate and idiosyncratic components. Standard principal analysis techniques, applied to a panel of sectors, can then be used recover idiosyncratic time series.

As an application, I employ the FIPE methodology in a Bayesian framework to estimate sectoral NKPCs for the US, Japan and the UK. I find that the pure-forward looking NKPC, with the real marginal cost proxied by the labor share, is rejected by the data.

${ }^{16}$ Batini et al. (2005) also estimate the slope of the Phillips curve for the UK economy. 


\section{References}

Bai J., Ng S. (2002), Determining the number of factors in approximate factor models, Econometric 70, pp. 191-221.

Boivin J., Giannoni M. P., Mihov I. (2009), Sticky prices and Monetary Policy: Evidence from Disaggregatd US Data, American Economic Review 99, pp. 350-384.

Basu S., Fernald J.G., Kimball M. S. (2006), Are Technology Improvements Contractionary? American Economic Review, 96, pp.1418-1448.

Batini N., Jackson B., Nickell S. (2005), An open-economy new Keynesian Phillips cirve for the UK, Journal of Monetary Economics 52, pp. 1061-1071.

Carvalho C. (2006), Heterogeneity in Price Stickiness and the Real Effect of Monetary Shocks, Frontiers of Macroeconomics vol. 2,issue 1, pp. 1-55.

Clarida R., Galií J., Gertler M. (1999), The Science of Monetary Policy: A New Keynesian Perspective, Journal of Economic Literature 37, pp. 16611707.

Cogley T., Sbordone A. M. (2008), Trend Inflation, Indexation, and Inflation Persistence in the New Keynesian Phillips Curve, American Economic Review 98, pp. 2101-21026.

Dennis R. (2006), The Frequency of Price Adjustment and New Keynesian Business Cycle Dynamics, Federal Reserve Bank of San Francisco Working Paper Series 2006-22.

Fuhrer J. C. (1997), The (Un)Importance of Forward-Looking Behavior in Price Specifications, Journal of Money, Credit and Banking 29, pp. 338-350.

Fuhrer J.C, Moore G. (1995), Inflation persistence, QUarterly Journal of Economics 110, pp. 127-159.

Gali J., Gertler M. (1999), Inflation dynamics: a structural econometric analysis, Journal of Monetary Economics 44, pp. 195-222.

Gali J., Gertler M., Lopez-Salido D. (2005), Robustness of the estimates of the hybrid New-Keynesian Phillips curve, Journal of Monetary Economics 52, pp. 1107-1118.

Klenow P. J., Kryvtsov (2008), State-Dependent and Time-Dependent Pricing: Does it Matter for Recent U.S. Inflation, Quarterly Journal of Economics 123, 863-904. 
Lindé J. (2005), Estimating New-Keynesian Phillips Curves: a full information maximum likelihood approach, Journal of Monetary Economics 52, pp. 1135-1149.

Maćkowiak B., Moench E., Wiederholt M. (2009), Sectoral price data and models of price setting, Journal of Monetary Economics 56, pp. 578-599.

Maćkowiak B., Wiederholt M. (2009), Optimal Sticky Prices under Rational Inattention, Ameican Economic Review 99, 769-803.

Mavroedis S. (2004), Identification Issues in Forward-Looking Models Estimated by GMM, with an Application to the Phillips Curve, Journal of Money, Credit and Banking 37, pp. 421-48.

Mavroedis S., Plagborg-Moller M., Stocks J.H. (2014), Empirical Evidence on Inflaton Expectations in the New Keynesian Phillips Curve, Journal of Economic Literature 52, pp. 124-188.

Melosi L. (2014), Estimating models with dispersed information, American Economic Journal: Macroeconomis 6, pp. 1-31.

Nakamura E., Steinsson J. (2008), Five Facts About Prices: A Reevaluation of Menu Cost Models, Quarterly Journal of Economics 123, 1415-1464.

Nakamura E., Steinsson J. (2013), Price Rigidity: Microeconomic Evidence and Macroeconomic Implications, Annual Review of Economics, Annual Reviews 5, pp. 133-163.

Pasaran M. H. (2006), Estimation and Inference in Large Heterogeneous Panels with a Multifactor Error Structure, Econometrica 74, pp. 967-1012.

Roberts J.M. (1995), The New Keynesian Economics and the Phillips Curve, Journal of Money, Credit and Banking 27, pp. 975-984.

Roberts J.M. (2005), How Well Does the New Keynesian Sticky-Price Model Fit the Data?, B. E. Journal of Macroeconomics: Contributions to Macroeconomics 5 .

Rudd, Whelan (2005), New tests of the New Keynesian Phillips Curve, Journal of Monetary Economics 52, pp. 1167-1181.

Rotemberg J. J., Woodford M. (1999), The Cyclical Behavior of Prices and Costs, Handbook of Macroeconomics, Taylor, J.B., and M. Woodford, eds., Amsterdam: North-Holland.

Sbordone A.M. (2002), Prices and unit labor costs: a new test of price sticki- 
ness, Journal of Monetary Economics 49, pp. 265292.

Schorfheide F. (2008), DSGE Model-Based Estimation of the New Keynesian Phillips Curve, Federal Reserve Bank of Richmond Economic Quarterly 94, pp. 397433.

Yun T. (1996), Nominal price rigidity, money supply endogeneity, and business cycles, Journal of Monetary Economics 37, pp. 345-370. 


\section{A Sectoral Phillips curves}

In every time $t$ firms in sector $i$ that can freely change their price will all choose the same $(\log )$ value $\bar{p}_{t}^{i}$ that solves the following first order condition

$$
E_{t} \sum_{s=0}^{\infty} \theta_{i}^{s} e^{\tilde{y}_{t+s}(i, j)+\bar{p}_{t}+\bar{\pi}_{t, t+s}^{i}-i_{t, t+s}}\left[1-e^{\tilde{\mu}_{t+s}^{i}+m_{t+s}^{n i}-\bar{p}_{t}^{i}-\bar{\pi}_{t, t+s}^{i}}\right]=0
$$

where $i_{t, t+s}$ and $\bar{\pi}_{t, t+s}^{i}$ are, respectively, the (log) cumulative nominal interest rate and cumulative growth of sectoral indexed prices between time $t$ and time $t+s$, so that $i_{t, t}=\bar{\pi}_{t, t}^{i}=0$. In particular, for $s \geq 1$ cumulative inflation indexation is implicitly defined by

$$
\bar{\pi}_{t, t+1+s}^{i}=\bar{\pi}_{t+1}^{i}+\bar{\pi}_{t+1, t+1+s}^{i}
$$

with as usual $\bar{\pi}_{t}^{i}$ indicating time $t$ growth of indexed prices.

Condition (28) guarantees that the expected discounted sum of marginal profit equals zero for any firm which freely resets its price at $t$. This condition can be linearized around an appropriate non-stochastic steady state with the property that for each sector $i$ firms that are allowed to reset choose the same $\log$ price $\bar{p}_{t}^{i}$ as firms that are not allowed to reset. This is a consequence of the assumption that $\bar{\pi}_{t}$ is a reasonable indexation variable for $\pi_{t}^{i}$, so that at a non-stochastic steady state $\pi^{i}=\bar{\pi}^{i}$. It follows that at such steady state $\pi_{t}^{i}=\bar{\pi}_{t}^{i}$ and $\tilde{y}_{t}(i, j)=y_{t}(i)$ for all $j$. Since $\bar{\nu}=1$, the sectoral aggregator has unit elasticity and thus, total $(\log )$ revenues are the same across sectors and grow at a constant aggregate value $\Delta Y+\pi$. Hence, normalizing time $t \log$ revenues $Y_{t}+P_{t}=0$, we have that at the steady state

$$
\tilde{y}_{t+s}(i, j)+\bar{p}_{t}+\bar{\pi}_{t, t+s}^{i}-i_{t, t+s}=s(\Delta Y+\pi-i)=s(\Delta Y-r) \equiv s \log \beta
$$

where $i$ and $r$ are, respectively, the $\log$ nominal and real steady state interest rate. As usual we take $r>\Delta Y$ and hence $\beta \in(0,1)$. Finally, linearizing around the steady state conditions $m_{t+s}^{n i}+\tilde{\mu}_{t+s}^{i}=p_{t}(i)$ and $\bar{p}_{t}^{i}+\bar{\pi}_{t, t+s}^{i}=p_{t}(i)$ we obtain

$$
E_{t} \sum_{s=0}^{\infty}(\beta \theta)^{s}\left[\tilde{\mu}_{t+s}^{i}+m_{t+s}^{n i}-\bar{p}_{t}^{i}-\bar{\pi}_{t, t+s}^{i}\right]=0
$$

or,

$$
\bar{p}_{t}^{i}=(1-\beta \theta) E_{t} \sum_{s=0}^{\infty}(\beta \theta)^{s}\left[\tilde{\mu}_{t+s}^{i}+m_{t+s}^{n i}-\bar{\pi}_{t, t+s}^{i}\right]
$$

By the law of iterated expectation and using (29) we have

$$
\bar{p}_{t}^{i}=\left(1-\beta \theta_{i}\right)\left[\log \mu_{i}+\tilde{m}_{t}(i, j)\right]+\beta \theta_{i} E_{t}\left[\bar{p}_{t+1}^{i}-\bar{\pi}_{t+1}^{i}\right]
$$

The $\log$ inflation rate for sector $s$ is

$$
\pi_{t}^{i}=\theta_{i} \bar{\pi}_{t}^{i}+\left(1-\theta_{i}\right)\left[\bar{p}_{t}^{i}-p_{t-1}(i)\right]
$$

We can then derive the sector specific forward-looking Phillips curve (1). 


\section{B Proof of Propositions}

Proof of Proposition 1. We calculate the form of the impulse responses to aggregate and idiosyncratic shock by considering the path of the real marginal cost gap in a perfect-forecast equilibrium. Combining (4) with (6), and substituting into (1) we obtain, after some algebra, the following second order difference equation

$$
\hat{m}_{t+1}^{r i}-\chi_{1} \hat{m}_{t}^{r i}+\chi_{0} \hat{m}_{t-1}^{r i}=\sum_{k=1}^{K_{m}} \hat{\psi}_{i k} q_{k t}+g_{t}^{i}
$$

for some set $K_{m}$ of aggregate factors $\left\{q_{k t}\right\}$ and corresponding sectoral loadings $\hat{\psi}_{i k}$, while $g_{i}(t)$ is defined as

$$
g_{i t}=\delta_{i}\left(\Delta \omega_{t+1}^{i}-\chi_{0} \Delta \omega_{t}^{i}\right)-\left(\Delta z_{t+1}^{i}-\chi_{0} \Delta z_{t}^{i}\right)+\left(\Delta \mu_{t+1}^{i}-\chi_{0} \Delta \mu_{t}^{i}\right)
$$

The impulse responses to shocks to the aggregate factors are easily obtained by focusing on the case $g_{i t}=0$. Assume that the process $\hat{x}_{t}=\sum_{K_{m}} \hat{f}_{k t}$ solves the equation

$$
\hat{x}_{t+1}-\chi_{1} \hat{x}_{t}+\chi_{0} \hat{x}_{t-1}=\sum_{k=1}^{K_{m}} q_{k t}
$$

for a set of $K_{m}$ aggregate factors $\hat{f}_{k t}$, where the factor $\hat{f}_{k t}$ gives the response to the corresponding factor $q_{k t}$. Then it is easy to see that $\hat{x}_{t}^{i}$ defined in (15) solves (32) for $g_{i}(t)=0$. The extrinsic persistence of the marginal cost gap to an aggregate structural shock, e.g. to aggregate productivity $Z_{t}$, is defined as the persistence of $\sum_{k} q_{k t}$ to such shock. In turn, the extrinsic persistence to an idiosyncratic shock is defined as the persistence of $g_{i t}$ to the shock.

We now derive the explicit form for the impulse response of to an idiosyncratic shock $\epsilon_{0} \equiv \epsilon_{0}^{i s} \iota_{s}$ at time zero and an initial condition $\hat{m}_{-1}^{i}=0$. The general solution to the non-homogeneous part of the difference equation (32) is characterized by two real roots with only one, $r \in(0,1)$, within the unit circle. Consider a particular and non explosive solution $\hat{m}_{t}^{p}$ to (32). The general solution to (32) is then

$$
\hat{m}_{t}^{i}=\epsilon_{0}^{i} A_{1}^{s} r^{t}+\hat{m}_{t}^{p}
$$

where $s \in\{\omega, z, \mu\}$ indicates that the constant $A_{1}^{s}$ depends on type of shock we are considering. Notice that we can write

$$
g_{i t+1}=\rho_{s} g_{i t} \quad t \geq 1
$$

with $\rho_{s}\left\{\rho_{\omega}, \rho_{z}, \rho_{\mu}\right\}$ depending on the type of shock we are considering. The expression above clarifies that the extrinsic persistence of an idiosyncratic shock $s$ equals $\rho_{s}$. It is easy to see that $g(1)=\left(1-\rho_{s}\right)\left(\chi_{0}-\rho_{s}\right) \epsilon_{0}$ and $g_{0}=-(1-$ $\left.\rho_{s}+\chi_{0}\right) \epsilon_{0}$. One can verify that for the following sequence of values $\hat{m}_{0}^{p}, \hat{m}_{1}^{p}, \ldots$ solves (32)

$$
\hat{m}_{t}^{p}=\epsilon_{0} \frac{\left(1-\rho_{s}\right)\left(\chi_{0}-\rho_{s}\right)}{\rho_{s}^{2}-\rho_{s} \chi_{1}+\chi_{0}} \rho_{s}^{t} \equiv \epsilon_{0} A_{2}^{s} \rho_{s}^{t}
$$


where we assume $\rho_{s}^{2}-\rho_{s} \chi_{1}+\chi_{0} \neq 0$. Using the initial condition $\hat{m}_{-1}=0$ we pin down the value of the constant $A_{1}^{i s}$ in (32) as

$$
A_{1}^{s}=-\frac{\chi_{0}\left(\chi_{1}-1-\chi_{0}\right)}{\left(\rho_{s}^{2}-\rho_{s} \chi_{1}+\chi_{0}\right)\left(\chi_{1}-r\right)}
$$

Summarizing, the solution for $t \geq 0$ to time zero impulses from the three idiosyncratic shocks is

$$
\hat{m}_{t}^{i}=\sum_{s \in\{\omega, z, \mu\}}\left(A_{1}^{s} r^{t}+A_{2}^{s} \rho_{s}^{t}\right) \epsilon_{0}^{s} \iota_{s}
$$

Summing all the past impacts of the shocks for all $j \geq 0$ we obtain equation (16), where clearly

$$
\mathrm{a}_{j}^{s}=A_{1}^{s} r^{j}+A_{2}^{s} \rho_{s}^{j}
$$

In an appendix available from the author it is shown that

$$
\mathrm{a}_{0}^{s} \equiv A_{1}^{s}+A_{2}^{s} \in(0,1)
$$

This concludes the proof that Assumption 1 is sufficient to allow a linear decomposition in of $\hat{m}_{t}^{r i}$ in aggregate and idiosyncratic parts. Necessity can be established with a counterexample. Consider a shock to an aggregate factor $q_{k t}$ that mimics the corresponding dynamics of $g_{i t}$ to one of the idiosyncratic shocks considered above. The impulse response of $\hat{m}_{t}^{r i}$ would be represented by the solution (35), with the idiosyncratic impulse $\epsilon_{0}^{s} \iota_{s}$ replaced by some aggregate impulse $\epsilon_{k 0}$. If Assumption 1 does not hold, then the root $r_{i}$ in such a solution would differ across sectors. Hence, the impulse responses of $\hat{m}_{t}^{r i}$ to a common aggregate shocks would follow paths characterized by different degrees $r_{i}$ of nonlinear decay, which could thus not be captured by a common non-linear decay $\hat{x}_{t}$.

Proof of Corollary 1. Assume that there are only aggregate shocks. Then, by iterating forward (1) and using (16), we obtain the inflation forecast error in (12) due to aggregate shocks as,

$$
e_{t+1}=\sum_{j=0}^{\infty} \sum_{k=1}^{K_{m}} \lambda_{i} \psi_{k i} \beta^{j} \underbrace{\left(E_{t}\left[\hat{f}_{k t}\right]-\hat{f}_{k t}\right)}_{\text {Factor }}
$$

Hence, since both $\hat{m}_{t}^{r i}$ and $\beta e_{t+1}$ can be expressed as a linear combination of aggregate factors, then the formulation $\tilde{x}_{t}^{i}$ is obtained.

Now consider the case with idiosyncratic shocks only. Use $\Delta m_{t}^{r i}$ in (6) to substitute $\Delta y_{t}(i)$ in (4). Then, substituting $\Delta m_{t}^{r i}$ with its corresponding idiosyncratic component $\Delta \hat{m}_{t}^{i}-\Delta \mu_{t}^{i}$, where $\Delta \hat{m}_{t}^{i}$ is obtained by differencing (16), then we have the expression for $e_{t+1}^{i}$ in (12).

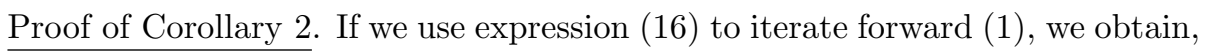

$$
\pi_{t}^{i}=\bar{\pi}_{t}^{i}+\lambda_{i} \sum_{s} \sum_{j=0}^{\infty} \alpha_{j}^{s} \epsilon_{t-j}^{i s}
$$


where $\alpha_{s}^{j}=\sum_{\tau=0}^{\infty} \beta^{\tau} \mathrm{a}_{j+\tau}^{s}$. Then, the $\mathrm{MA}(\infty)$ form for the inflation gap is

$$
\xi_{t}^{i}=\beta \epsilon_{t+1}^{i \gamma}-(1-\beta) \sum_{j=0}^{\infty} \epsilon_{t-j}^{i \gamma}-\beta \lambda_{i} \sum_{s} \alpha_{0}^{s} \epsilon_{t+1}^{i s}+\lambda_{i} \sum_{s} \sum_{j=0}^{\infty} \tilde{\alpha}_{j}^{s} \epsilon_{t-j}^{i s}
$$

with $\tilde{\alpha}_{j}^{s}=\alpha_{j}^{s}-\beta \alpha_{j+1}^{s}$. The $\mathrm{MA}(\infty)$ representation for $m_{t}^{i}$ is

$$
m_{t}^{i}=\sum_{s} \sum_{j=0}^{\infty} \tilde{\mathrm{a}}_{j}^{s} \epsilon_{t-j}^{i s} \iota_{s}
$$

with $\tilde{\mathrm{a}}_{j}^{s}=\mathrm{a}_{j}^{s}-\rho_{\mu}^{j}$ for $s=\mu$ and $\tilde{\mathrm{a}}_{j}^{s}=\mathrm{a}_{j}^{s}$ otherwise. Hence, for any given natural number $n=0,1, \ldots$, we obtain

$$
\begin{aligned}
\operatorname{Cov}\left(\xi_{t}^{i}, m_{t+n}^{i}\right) & =\lambda_{i} \sum_{s} \sigma_{s}^{2} \sum_{j=0}^{\infty} \tilde{\alpha}_{j}^{s} \tilde{\mathrm{a}}_{j+n}^{s}=\lambda_{i} \sigma^{2}\left[R \sum_{j=0}^{\infty} \tilde{\alpha}_{j}^{\mu} \tilde{\mathrm{a}}_{j+n}^{\mu}+(1-R) \sum_{j=0}^{\infty} \tilde{\alpha}_{j}^{\omega} \tilde{\mathrm{a}}_{j+n}^{\omega}\right] \\
\operatorname{Cov}\left(\xi_{t}^{i}, m_{t-n}^{i}\right) & =\lambda_{i} \sum_{s} \sigma_{s}^{2} \sum_{j=0}^{\infty} \tilde{\alpha}_{j+n}^{s} \tilde{\mathrm{a}}_{j}^{s}=\lambda_{i} \sigma^{2}\left[R \sum_{j=0}^{\infty} \tilde{\alpha}_{j+n}^{\mu} \tilde{\mathrm{a}}_{j}^{\mu}+(1-R) \sum_{j=0}^{\infty} \tilde{\alpha}_{j+n}^{\omega} \tilde{\mathrm{a}}_{j}^{\omega}\right] \\
\operatorname{Var}\left(m_{t}^{i}\right) & =\sum_{s} \sigma_{s}^{2} \sum_{j=0}^{\infty}\left(\tilde{\mathrm{a}}_{j}^{s}\right)^{2}=\sigma^{2}\left[R \sum_{j=0}^{\infty}\left(\tilde{\mathrm{a}}_{j}^{\mu}\right)^{2}+(1-R) \sum_{j=0}^{\infty}\left(\tilde{\mathrm{a}}_{j}^{\omega}\right)^{2}\right]
\end{aligned}
$$

with $\sigma^{2}=\sum_{s} \sigma_{s}^{2}$ and the second equality of each of the above equation using the fact that $\rho_{\omega}=\rho_{z}$ implies $\mathrm{a}_{j}^{\omega}=\mathrm{a}_{j}^{z}$. Recall that, for given values of $\beta$ and of the extrinsic persistences $\rho_{s}$, the weights $\mathrm{a}_{j}^{s}$ depend only on $r$. It then follows that the projection of $\xi_{t}^{i}$ on any lead or lag of $m_{t}^{i}$ is obtained as the product of $\lambda_{i}$ and of a function $b(r, R)$ explicitly obtained by taking the appropriate ratios of the expressions above. Finally, starting from the MA $(\infty)$ representations of $\xi_{t}^{i}$ and $m_{t}^{i}$, corresponding $\mathrm{MA}(\infty)$ representations for first-differenced variables $\Delta \xi_{t}^{i}$ and $\Delta m_{t}^{i}$ are easily derived. Repeating the steps above one can then shows that the required representation for the projections is again obtained.

\section{Moments}

Define $\iota_{\rho}$ an indicator function which equals 1 if $\rho=0$ and 0 if $\rho=1$ and define $\hat{\sigma}^{2}=\sigma_{\omega}^{2}+\sigma_{z}^{2}$. We express the moments in terms of $\hat{R}=\frac{R}{1-R}$.

We can write the process for $\Delta m_{t}^{i}$ as

$$
\begin{gathered}
\Delta m_{t}^{i}=r \Delta m_{t-1}^{i}+\Delta \varepsilon_{t}^{i} \\
\Delta \varepsilon_{t}^{i}=\left(\iota_{\rho}+r\right) \epsilon_{t-1}^{i \mu}-\epsilon_{t}^{i \mu}-\iota_{\rho} r \epsilon_{t-2}^{i \mu}+\Delta \epsilon_{t}^{i}-\frac{\iota_{\rho} r}{\mathrm{a}_{0}} \Delta \epsilon_{t-1}^{i} \\
\Delta \epsilon_{t}^{i}=\mathrm{a}_{0}\left(\Delta \epsilon_{t}^{i \omega}-\Delta \epsilon_{t}^{i z}+\Delta \epsilon_{t}^{i \mu}\right)
\end{gathered}
$$


Define the following moments for the errors,

$$
\begin{aligned}
\frac{\operatorname{Var}\left(\Delta \varepsilon_{t}^{i}\right)}{\hat{\sigma}^{2}} & =\zeta_{0}(r, \hat{R})=\mathrm{a}_{0}^{2}+\iota_{\rho} r^{2}+\left(\mathrm{a}_{0}+\iota_{\rho} r\right)^{2}+\left[\left(1-\mathrm{a}_{0}\right)^{2}+\left(\iota_{\rho}+r-\mathrm{a}_{0}-\iota_{\rho} r\right)^{2}\right] \hat{R} \\
\frac{\operatorname{Cov}\left(\Delta \varepsilon_{t}^{i}, \Delta \varepsilon_{t-1}^{i}\right)}{\hat{\sigma}^{2}} & =\zeta_{1}(r, \hat{R})=-\left[\left(\mathrm{a}_{0}+\iota_{\rho} r\right)^{2}+\left(\iota_{\rho}+r-\mathrm{a}_{0}-\iota_{\rho} r\right)\left(1-\mathrm{a}_{0}\right) \hat{R}\right] \\
\frac{\operatorname{Cov}\left(\Delta \varepsilon_{t}^{i}, \Delta \varepsilon_{t-2}^{i}\right)}{\hat{\sigma}^{2}} & =\zeta_{2}(r, \hat{R})=\mathrm{a}_{0} \iota_{\rho} r
\end{aligned}
$$

Also,

$$
\begin{aligned}
\frac{\operatorname{Cov}\left(\Delta u_{t}^{i}, \Delta \varepsilon_{t}^{i}\right)}{\lambda_{i} \hat{\sigma}^{2}} & =\tilde{\zeta}_{0}(r, \hat{R})=\frac{1-\mathrm{a}_{0}}{\chi_{0} \kappa}\left\{\mathrm{a}_{0}-\left[\chi_{0} \kappa+1-\mathrm{a}_{0}+\frac{\chi_{0} \kappa\left(\iota_{\rho}+r-\mathrm{a}_{0}-\iota_{\rho} r\right) \iota_{\rho}}{1-\mathrm{a}_{0}}\right] \hat{R}\right\} \\
\frac{\operatorname{Cov}\left(\Delta u_{t}^{i}, \Delta \varepsilon_{t-1}^{i}\right)}{\lambda_{i} \hat{\sigma}^{2}} & =\tilde{\zeta}_{1}(r, \hat{R})=\left(1-\mathrm{a}_{0}\right) \iota_{\rho} \hat{R} \\
\frac{\operatorname{Cov}\left(\Delta u_{t}^{i}, \Delta \varepsilon_{t+1}^{i}\right)}{\lambda_{i} \hat{\sigma}^{2}} & =\tilde{\zeta}_{-1}(r, \hat{R})=\frac{1-\mathrm{a}_{0}}{\chi_{0} \kappa}\left\{\left[1-\mathrm{a}_{0}+\left(\iota_{\rho}+r-\mathrm{a}_{0}-\iota_{\rho} r\right)\left(\frac{\chi_{0} \kappa}{1-\mathrm{a}_{0}}+1\right)\right] \hat{R}-2 \mathrm{a}_{0}-\iota_{\rho} r\right\} \\
\frac{\operatorname{Cov}\left(\Delta u_{t}^{i}, \Delta \varepsilon_{t+2}^{i}\right)}{\lambda_{i} \hat{\sigma}^{2}} & =\tilde{\zeta}_{-2}(r, \hat{R})=\frac{1-\mathrm{a}_{0}}{\chi_{0} \kappa}\left[2 \iota_{\rho} r+\mathrm{a}_{0}-\left(\iota_{\rho}+r-\mathrm{a}_{0}-\iota_{\rho} r\right) \hat{R}\right]
\end{aligned}
$$

No lagged values of $\Delta \xi_{t}^{i}$ as regressors. We can calculate the following moments,

$$
\begin{gathered}
\frac{\operatorname{Var}\left(\Delta m_{t}^{i}\right)}{\hat{\sigma}^{2}}=v(r, \hat{R})=\frac{\zeta_{0}(r, \hat{R})+2 r \zeta_{1}(r, \hat{R})+2 r^{2} \zeta_{2}(r, \hat{R})}{1-r^{2}} \\
\frac{\operatorname{Cov}\left(\Delta m_{t}^{i}, \Delta m_{t-1}^{i}\right)}{\operatorname{Var}\left(\Delta m_{t}^{i}\right)}=a_{1}(r, \hat{R})=r+\frac{\zeta_{1}(r, \hat{R})+r \zeta_{2}(r, \hat{R})}{v(r, \hat{R})} \\
\frac{\operatorname{Cov}\left(\Delta m_{t+1}^{i}, \Delta m_{t-1}^{i}\right)}{\operatorname{Var}\left(\Delta m_{t}^{i}\right)}=a_{2}(r, \hat{R})=r a_{1}(r, \hat{R})+\frac{\zeta_{2}(r, \hat{R})}{v(r, \hat{R})} \\
\frac{\operatorname{Cov}\left(\Delta \xi_{t}^{i}, \Delta m_{t-1}^{i}\right)}{\lambda_{i} \operatorname{Var}\left(\Delta m_{t}^{i}\right)}=b_{1}(r, \hat{R})=a_{1}(r, \hat{R})+\frac{\tilde{\zeta}_{1}(r, \hat{R})}{v(r, \hat{R})} \\
\frac{\operatorname{Cov}\left(\Delta \xi_{t}^{i}, \Delta m_{t}^{i}\right)}{\lambda_{i} \operatorname{Var}\left(\Delta m_{t}^{i}\right)}=b_{0}(r, \hat{R})=1+\frac{r \tilde{\zeta}_{1}(r, \hat{R})+\tilde{\zeta}_{0}(r, \hat{R})}{v(r, \hat{R})} \\
\frac{\operatorname{Cov}\left(\Delta \xi_{t}^{i}, \Delta m_{t+1}^{i}\right)}{\lambda_{i} \operatorname{Var}\left(\Delta m_{t}^{i}\right)}=b_{-1}(r, \hat{R})=a_{1}(r, \hat{R})+\frac{r^{2} \tilde{\zeta}_{1}(r, \hat{R})+r \tilde{\zeta}_{0}(r, \hat{R})+\tilde{\zeta}_{-1}(r, \hat{R})}{v(r, \hat{R})} \\
\frac{\operatorname{Cov}\left(\Delta \xi_{t}^{i}, \Delta m_{t+2}^{i}\right)}{\lambda_{i} \operatorname{Var}\left(\Delta m_{t}^{i}\right)}=b_{-2}(r, \hat{R})=r b_{-1}(r, \hat{R})+\frac{\zeta_{2}(r, \hat{R})+\tilde{\zeta}-2(r, \hat{R})}{v(r, \hat{R})}
\end{gathered}
$$

The variance covariance matrix $\Sigma_{a}$ is then given by

$$
\Sigma_{a}=\left[\begin{array}{ccc}
1 & a_{1}(r, \hat{R}) & a_{2}(r, \hat{R}) \\
a_{1}(r, \hat{R}) & 1 & a_{1}(r, \hat{R}) \\
a_{2}(r, \hat{R}) & a_{1}(r, \hat{R}) & 1
\end{array}\right]
$$


The vector $\Sigma_{b}$ is simply $\Sigma_{b}=\left[b_{1}(r, \hat{R}) \quad b_{0}(r, \hat{R}) \quad b_{-1}(r, \hat{R})\right]$.

Inclusion of lagged values of $\Delta \xi_{t}^{i}$ as regressors. As done for $\hat{R}$, we can express the moment conditions as a function of the transformed variable $\hat{\Gamma}=\Gamma /(1-\Gamma)$. In our empirical specification we set $J=1$, i.e. only one lag of $\delta \xi_{t}^{i}$ is included in $X_{t}^{i}$. We then compute the following quantities,

$$
\begin{gathered}
\frac{\operatorname{Var}\left(\Delta u_{t}^{i}\right)}{\lambda_{i}^{2} \hat{\sigma}^{2}}=\hat{\zeta}_{0}(r, \hat{R}, \hat{\Gamma})=2\left[\left(\frac{1+\iota_{\rho}}{2}+\frac{1-\mathrm{a}_{0}}{\chi_{0} \kappa}\right) \hat{R}+\frac{\left(1-\mathrm{a}_{0}\right)^{2}}{\chi_{0}^{2} \kappa^{2}}(1+\hat{R})(1+\hat{\Gamma})\right] \\
\frac{\operatorname{Cov}\left(\Delta u_{t}^{i}, \Delta u_{t-1}^{i}\right)}{\lambda_{i}^{2} \hat{\sigma}^{2}}=\hat{\zeta}_{1}(r, \hat{R}, \hat{\Gamma})=-\left\{\left[\frac{\left(1+\iota_{\rho}\right)\left(1-\mathrm{a}_{0}\right)}{\chi_{0} \kappa}+\iota_{\rho}\right] \hat{R}+\frac{\left(1-\mathrm{a}_{0}\right)^{2}}{\chi_{0}^{2} \kappa^{2}}(1+\hat{R})(1+\hat{\Gamma})\right\}
\end{gathered}
$$

The greater the value of $\hat{\Gamma}$, the greater the contribution of "measurement error" to the variability of the regression error $\Delta u_{t}^{i}$ and to its (negative) autocovariance. We then have,

$$
\begin{gathered}
\frac{\operatorname{Var}\left(\Delta \xi_{t-1}^{i}\right)}{\lambda_{i}^{2} \operatorname{Var}\left(\Delta m_{t}^{i}\right)}=\hat{a}_{0}(r, \hat{R}, \hat{\Gamma})=b_{0}(r, \hat{R})+\frac{r \tilde{\zeta}_{1}(r, \hat{R})+\tilde{\zeta}_{0}(r, \hat{R})+\hat{\zeta}_{0}(r, \hat{R}, \hat{\Gamma})}{v(r, \hat{R})} \\
\frac{\operatorname{Cov}\left(\Delta \xi_{t}^{i}, \Delta \xi_{t-1}^{i}\right)}{\lambda_{i}^{2} \operatorname{Var}\left(\Delta m_{t}^{i}\right)}=\hat{b}_{1}(r, \hat{R}, \hat{\Gamma})=b_{1}(r, \hat{R})+\frac{r^{2} \tilde{\zeta}_{1}(r, \hat{R})+r \tilde{\zeta}_{0}(r, \hat{R})+\tilde{\zeta}_{-1}(r, \hat{R})+\hat{\zeta}_{1}(r, \hat{R}, \hat{\Gamma})}{v(r, \hat{R})}
\end{gathered}
$$

The covariance matrix $\Sigma_{a}$ under $X_{t}^{i}$ is

$$
\Sigma_{a}=\left[\begin{array}{cccc}
\lambda_{i}^{2} \hat{a}_{0}(r, \hat{R}, \hat{\Gamma}) & \lambda_{i} b_{0}(r, \hat{R}) & \lambda_{i} b_{-1}(r, \hat{R}) & \lambda_{i} b_{-2}(r, \hat{R}) \\
\lambda_{i} b_{0}(r, \hat{R}) & \ddots & \vdots & . \\
\lambda_{i} b_{-1}(r, \hat{R}) & \ldots & \Sigma_{a} & \ldots \\
\lambda_{i} b_{-2}(r, \hat{R}) & . & \vdots & \ddots
\end{array}\right]
$$

\section{Prior and posteriors for $r, R, \Gamma$}

This section provides more details on prior and posterior densities.

\section{D.1 Priors}

Priors are independent from each other. The prior for $\mu$ is assumed to be a normal with mean zero and variance equal to 9 . For the other parameters, we rewrite the moments conditions in terms of auxiliary monotonic transformations, over which we postulate our priors. In particular, for $r$ and $R$ we use the auxiliary parameters $\hat{r}=\frac{r}{1-r}$ and $\hat{R}=\frac{R}{1-R}$, respectively. Given our restrictions on the original parameters, the transformed ones span the positive real line and their prior is taken to be a gamma distribution. For $\hat{r}$, the shape parameter of the distribution is 4 and the scale is .25, while for $R$ the scale is 2 and the shape is again 4 . 
The prior for $\Gamma$ is dealt with in a slightly different way. Notice that, when the forward-looking Phillips curve fails because $\lambda_{i}=0$, then the covariance matrix $\Sigma_{a}$ is singular. To avoid this situation, in our estimation implementation we write the moment conditions as functions of $\hat{\Gamma}=\lambda_{i}^{2} \frac{\Gamma}{1-\Gamma)}$, which is the usual transformed parameters scaled by $\lambda$. We assume a uniform prior for $\hat{\Gamma}$ on the range $[0,2]$. In terms of interpretation, if $\lambda_{i} \rightarrow 0$ and $\hat{\Gamma}>0$, then $\Gamma /(1-\Gamma)=\infty$ and thus $\Gamma=1$. This implies that all the variability of $\Delta e_{t}^{i}$ is due to variation in the normalized measurement error $\Delta \tilde{\epsilon}_{t}^{i \gamma}=\Delta \epsilon^{i \gamma} / \lambda_{i}$, a result that requires $\operatorname{Var}\left(\Delta \tilde{\epsilon}_{t}^{i \gamma}\right)=\infty$. This naturally obtains as a consequence of $\operatorname{Var}\left(\epsilon_{t}^{i \gamma}\right)>0$ and $\lambda_{i} \rightarrow 0$. Conversely, if $\bar{\lambda}=2$, then the uniform prior implies that $\Gamma$ has an upper bound of $2 / 3$, which means that that the normalized measurement error can account for at most $2 / 3$ of the variability in the inflation forecast error. 


\section{D.2 Posteriors}

The implied posterior distributions for the original parameters and $r$ and $R$, and for the transformed $\hat{\Gamma}$ are the following.
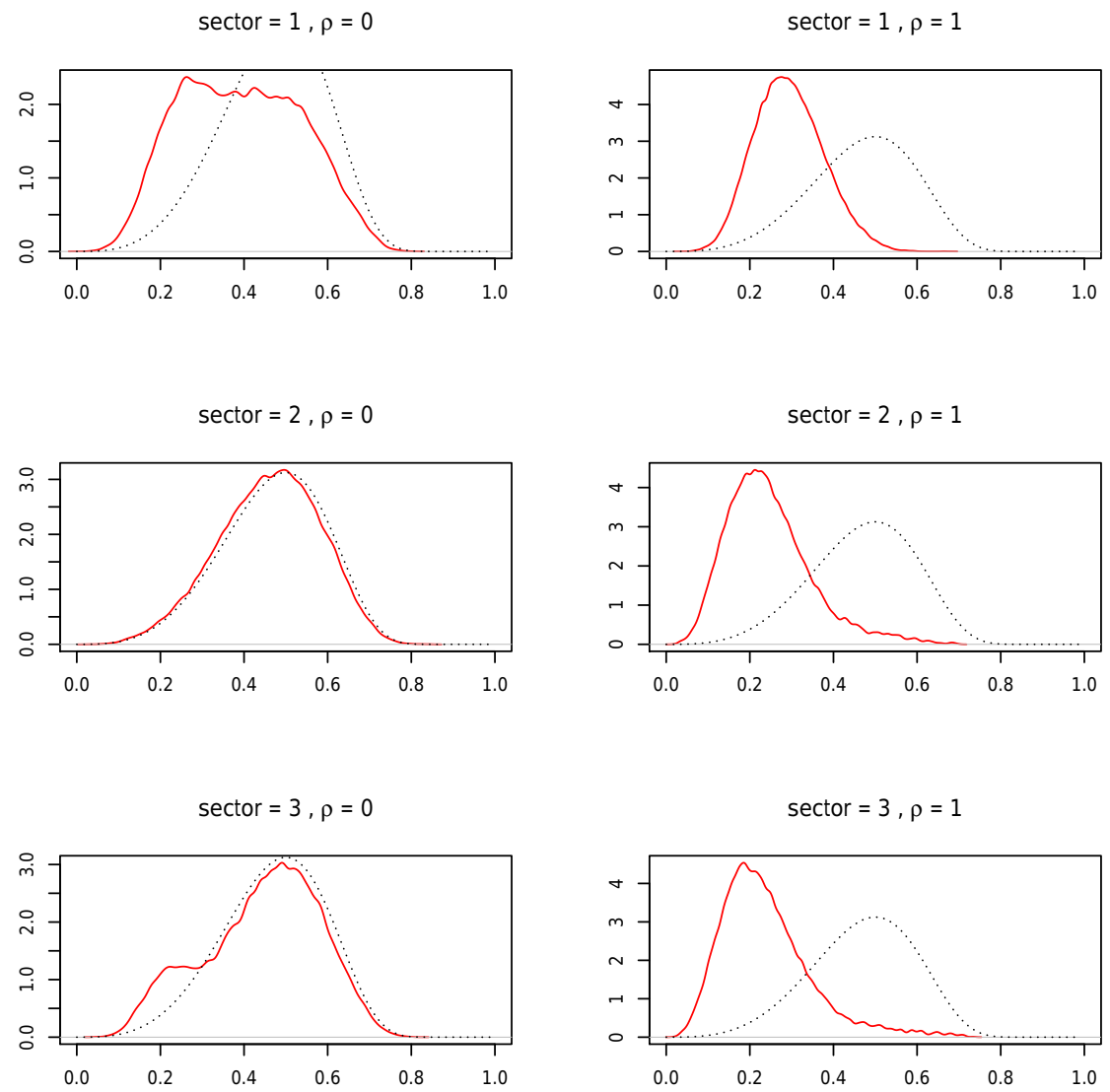

Figure 5: Smoothed posterior density of $r$ for the US. Dotted lines indicate prior densities. 

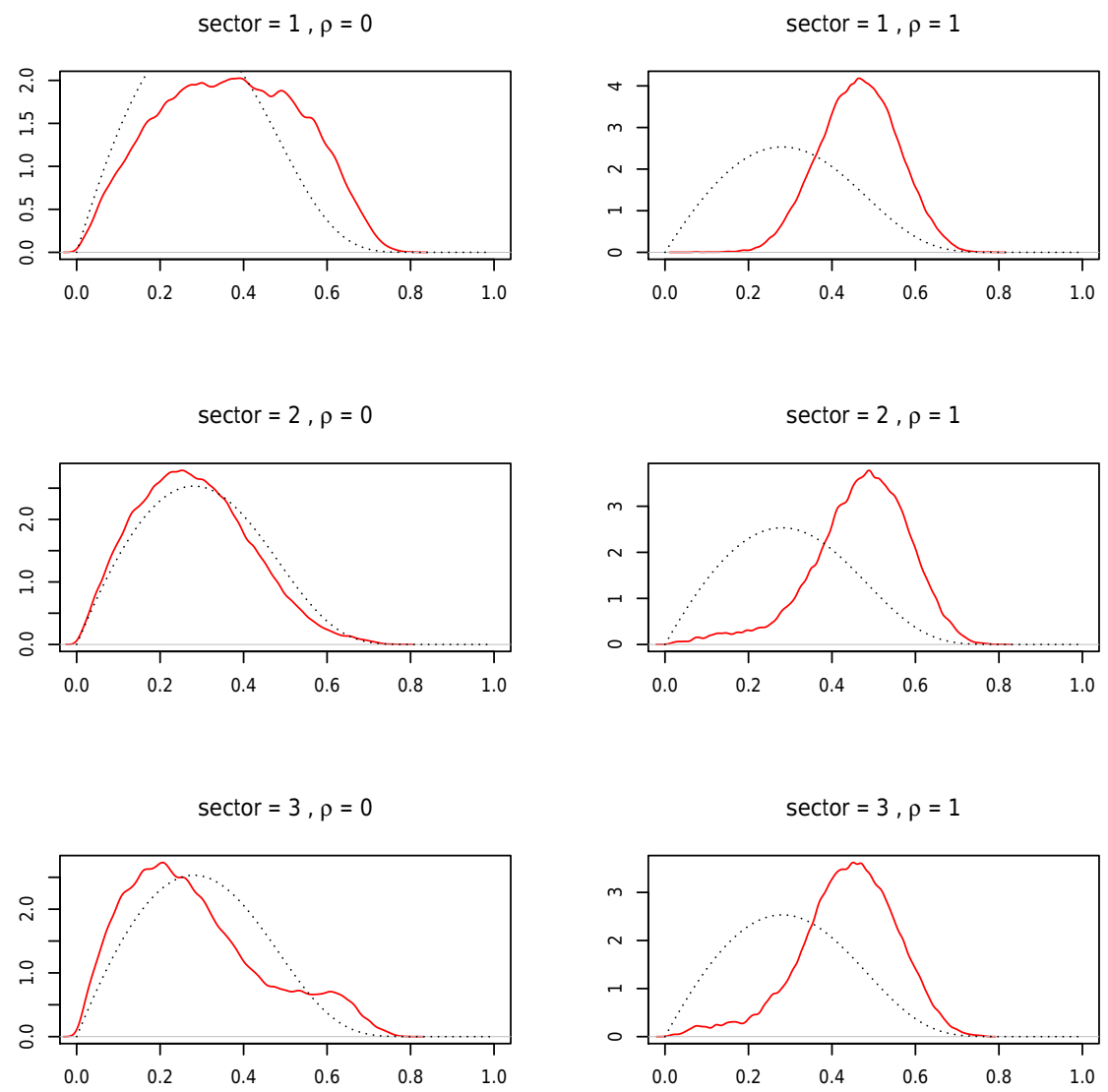

Figure 6: Smoothed posterior density of $R$ for the US. Dotted lines indicate prior densities. 

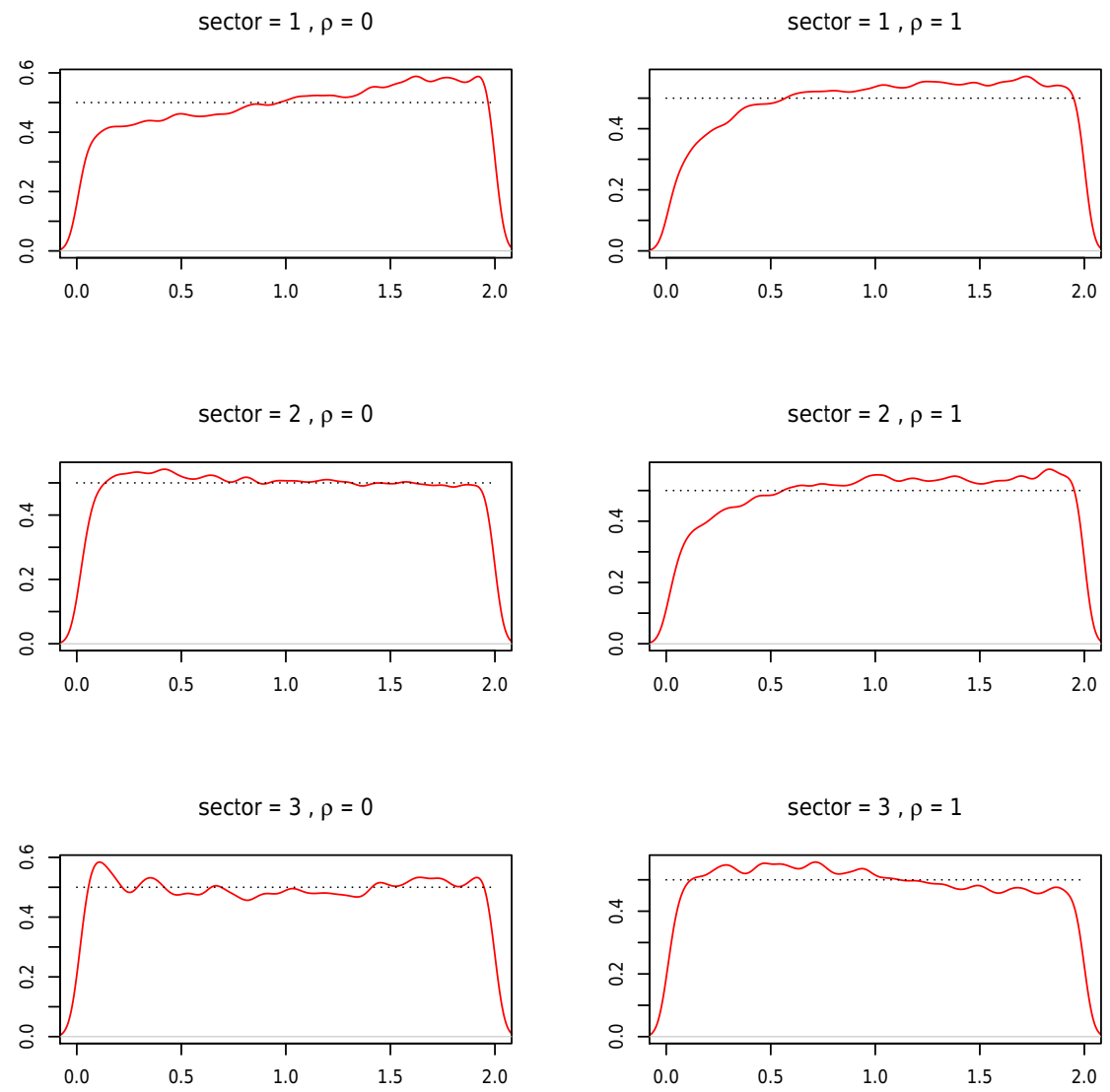

Figure 7: Smoothed posterior density of $\hat{\Gamma}$ for the US. Dotted lines indicate prior densities. 

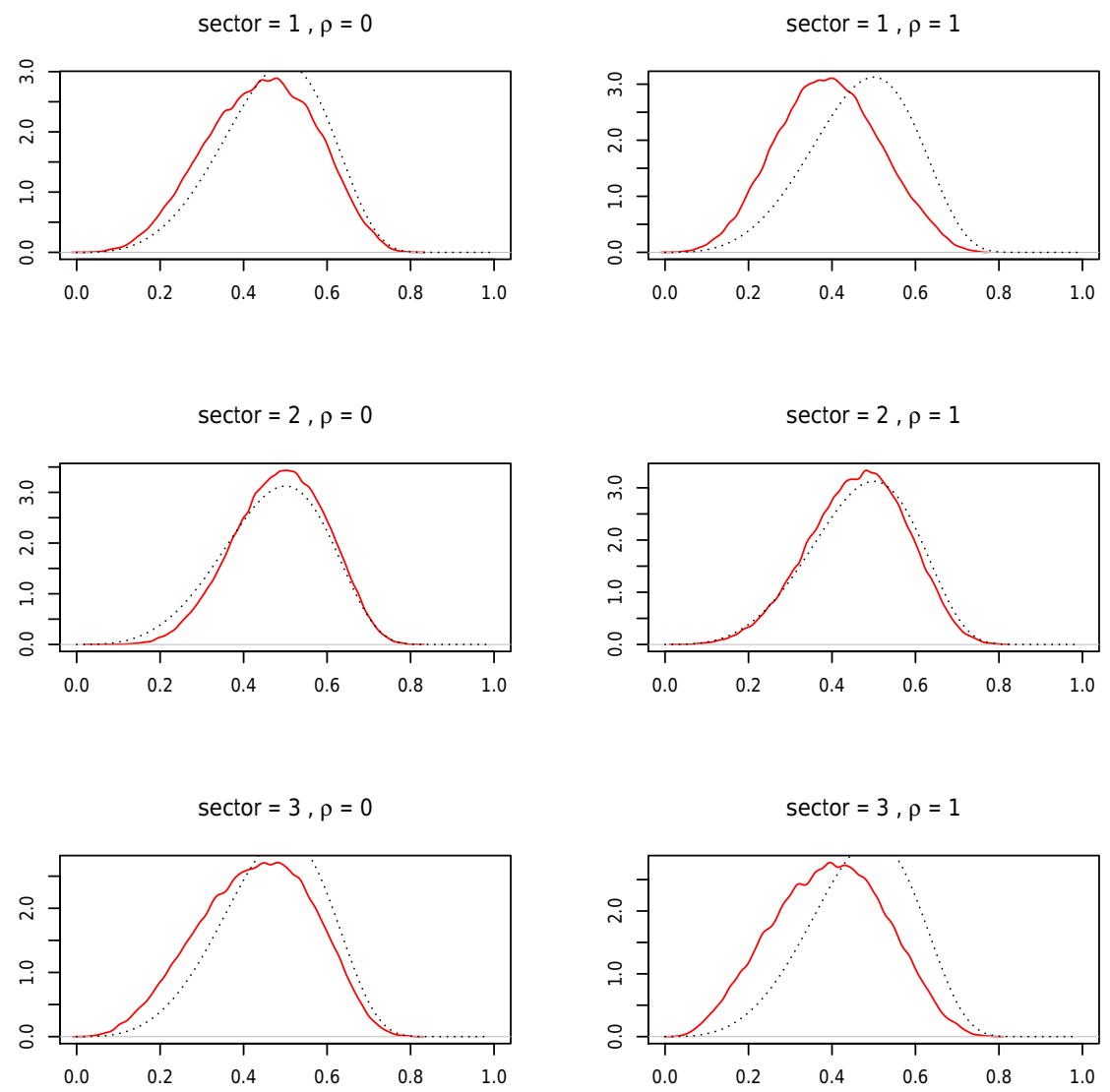

Figure 8: Smoothed posterior density of $r$ for Japan. Dotted lines indicate prior densities. 

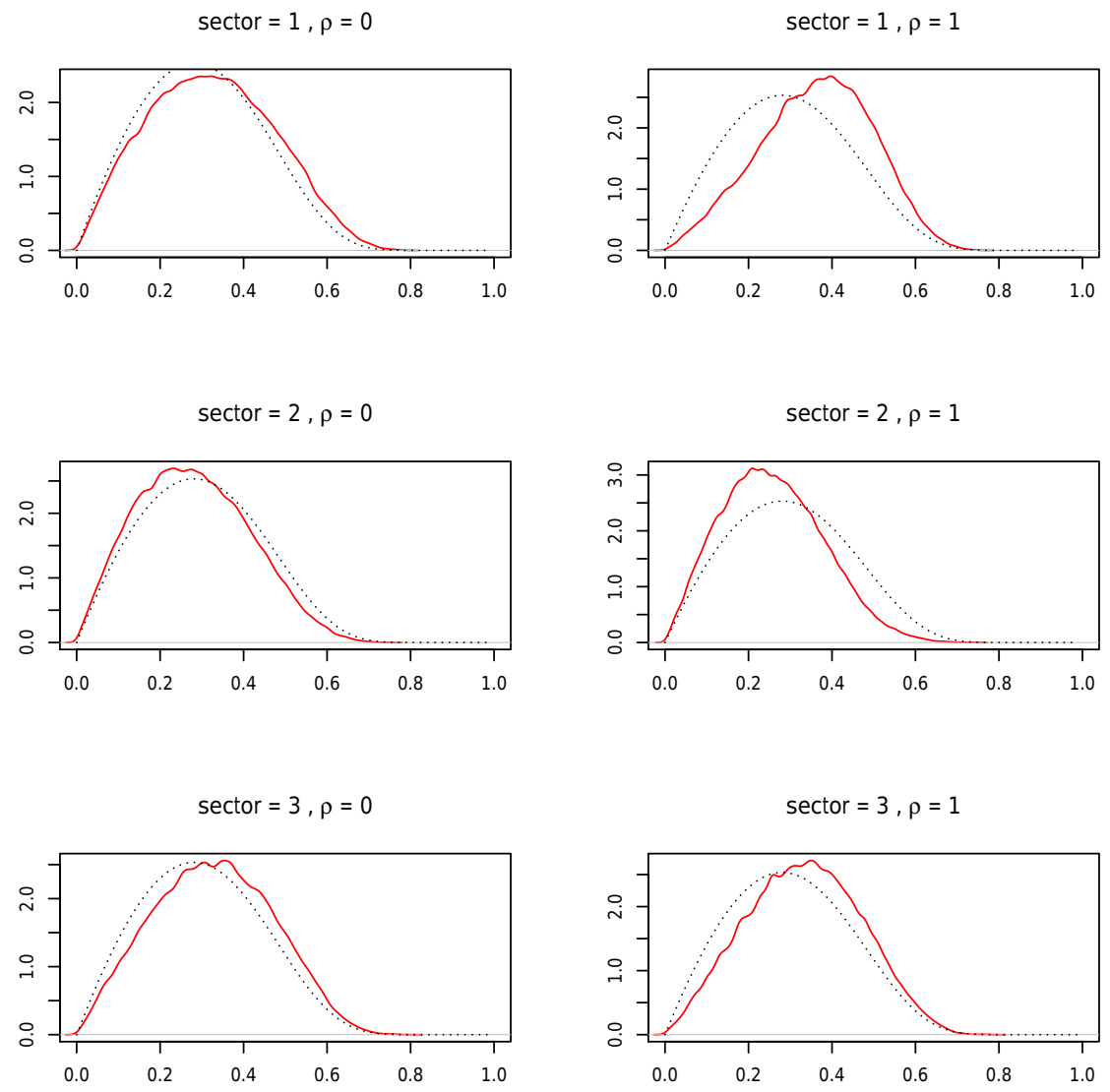

Figure 9: Smoothed posterior density of $R$ for Japan. Dotted lines indicate prior densities. 

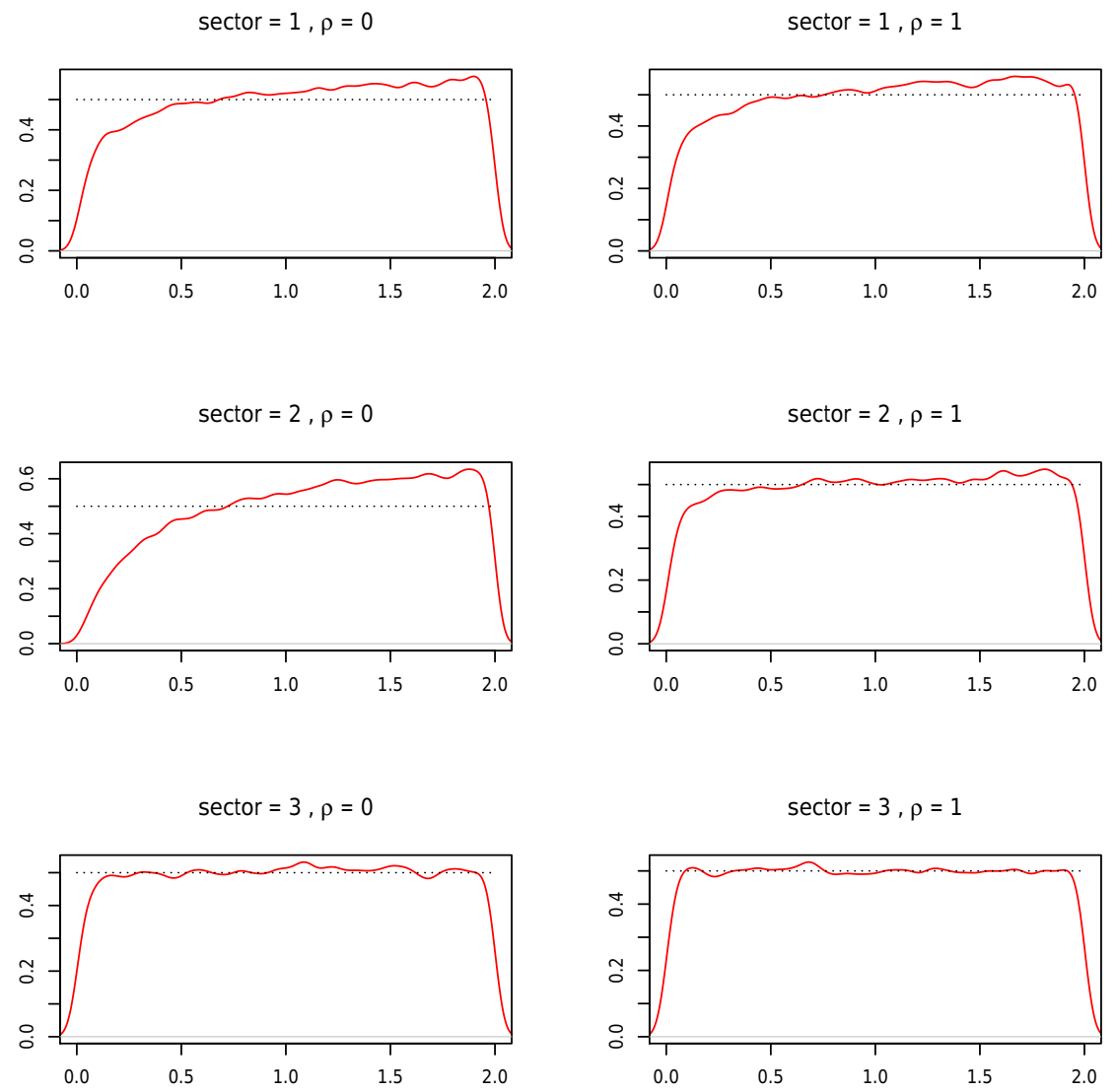

Figure 10: Smoothed posterior density of $\hat{\Gamma}$ for Japan. Dotted lines indicate prior densities. 

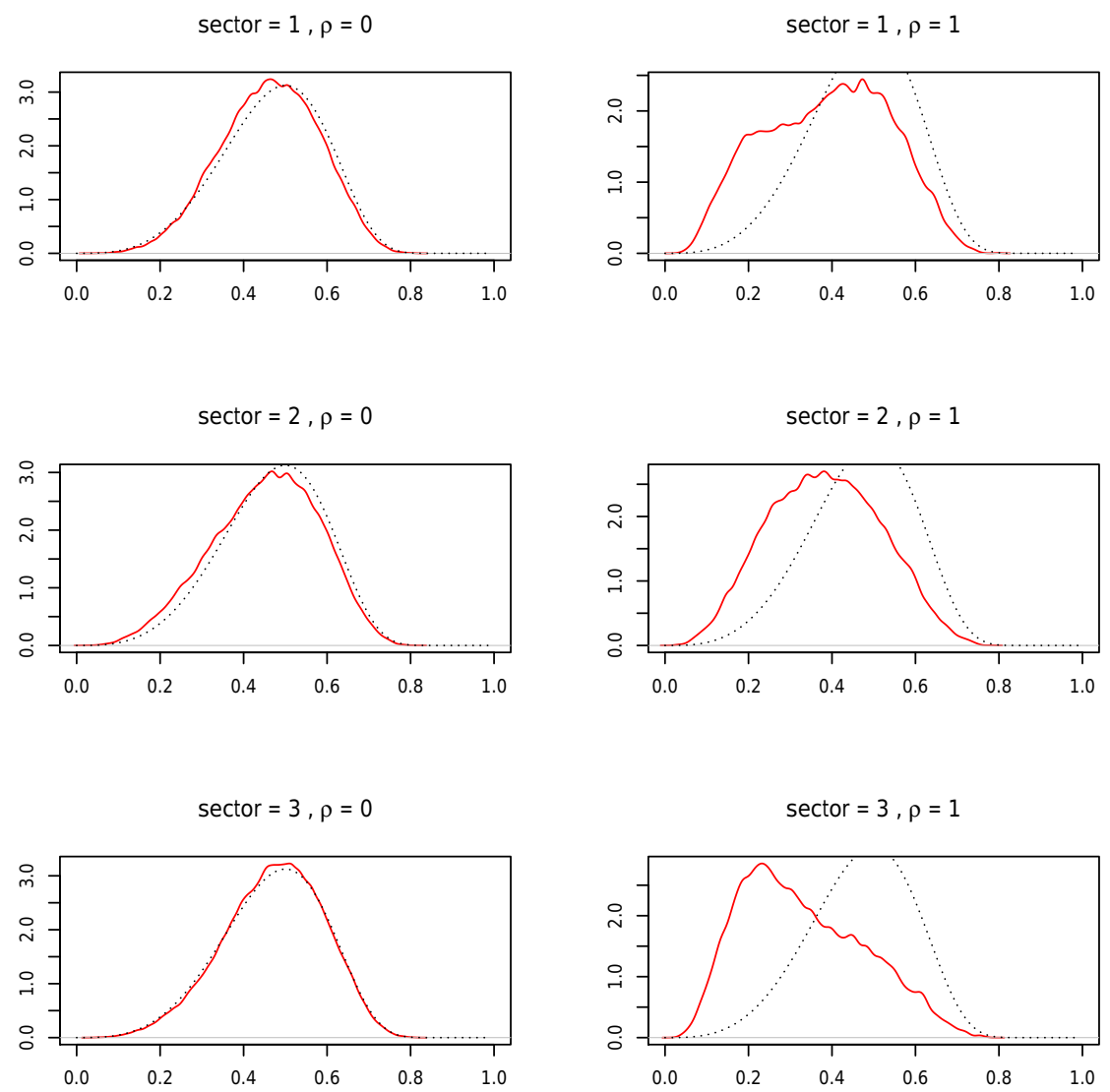

Figure 11: Smoothed posterior density of $r$ for the UK. Dotted lines indicate prior densities. 

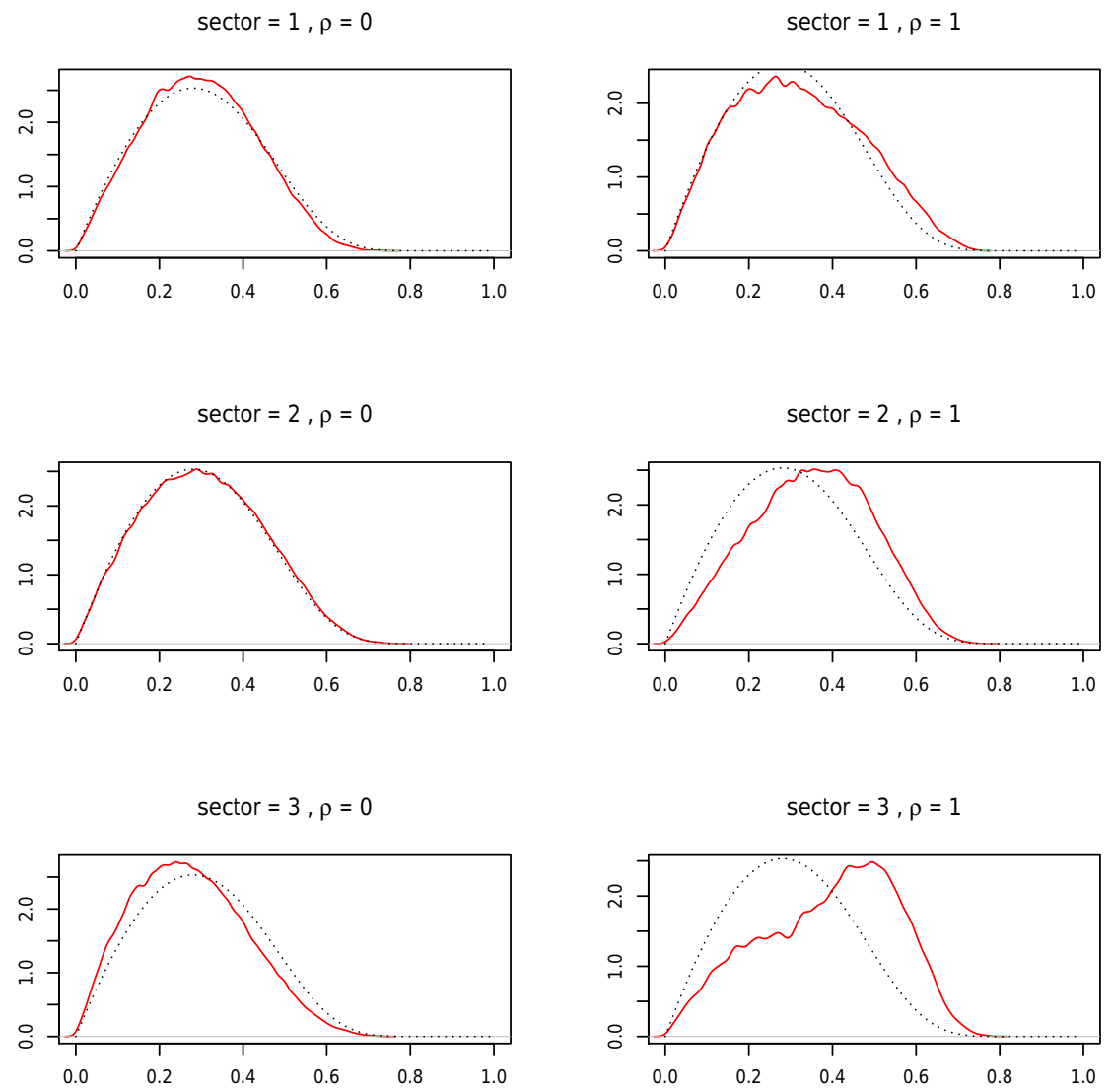

Figure 12: Smoothed posterior density of $R$ for the UK. Dotted lines indicate prior densities. 

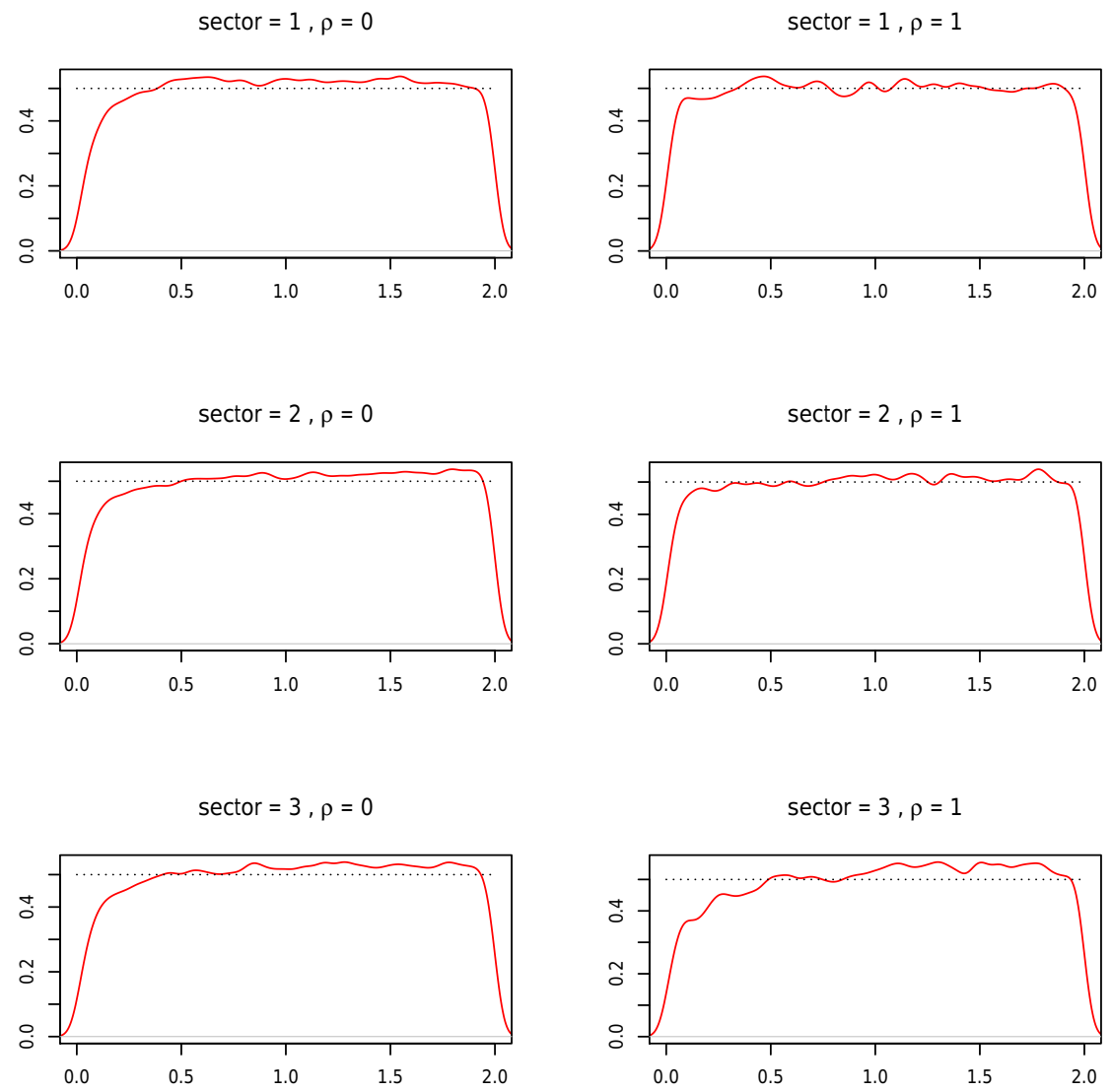

Figure 13: Smoothed posterior density of $\hat{\Gamma}$ for the UK. Dotted lines indicate prior densities. 


\section{D.3 Posteriors diagnostics}

In all the estimations, the rejection rates from the proposal are $24 \%$. The following figures report the sample auto-correlations of the parameters draws.
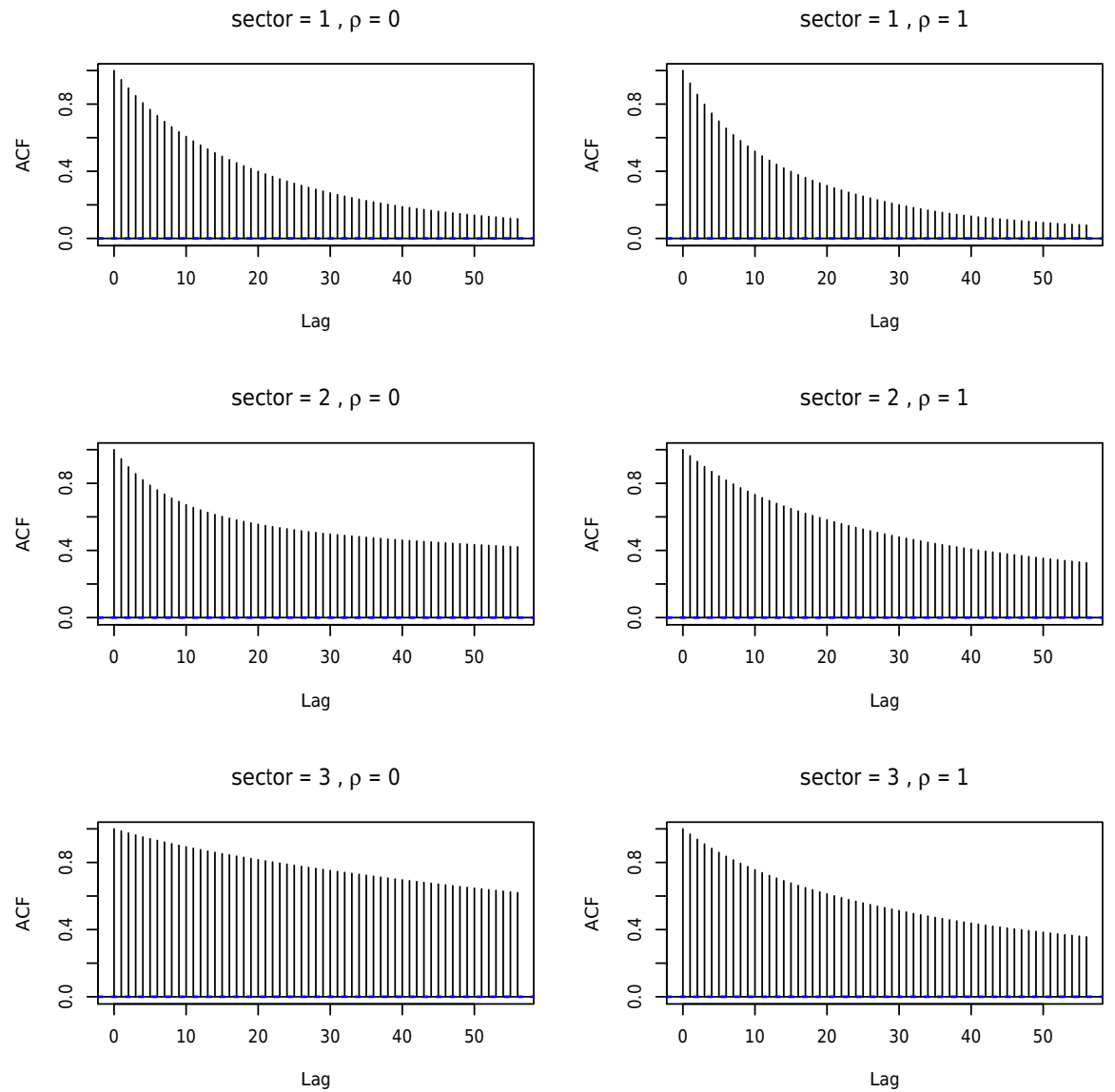

Figure 14: Auto-correlation function of draws for $\bar{\lambda}$ and the US. 

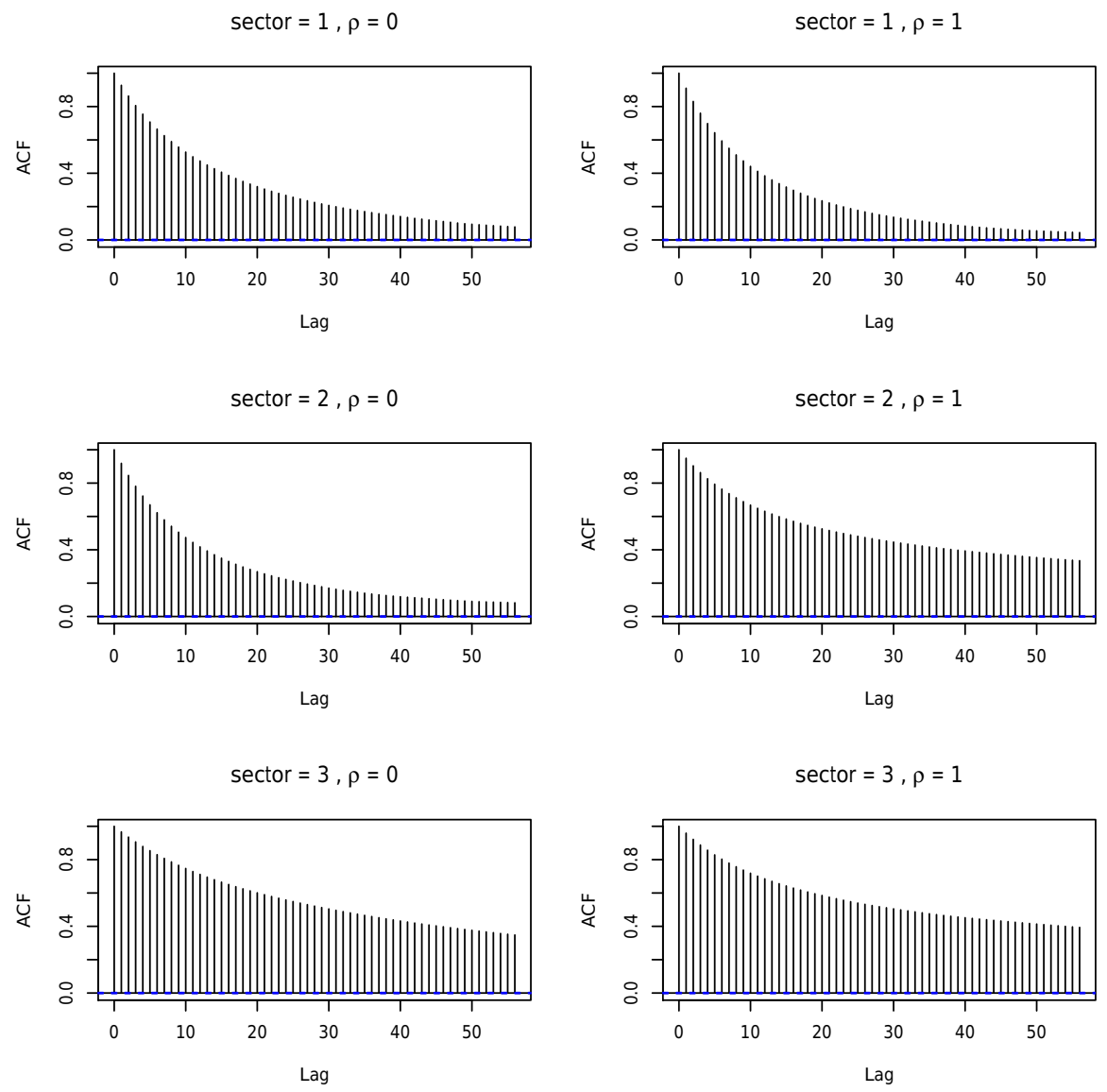

Figure 15: Auto-correlation function of draws for $r$ and the US. 

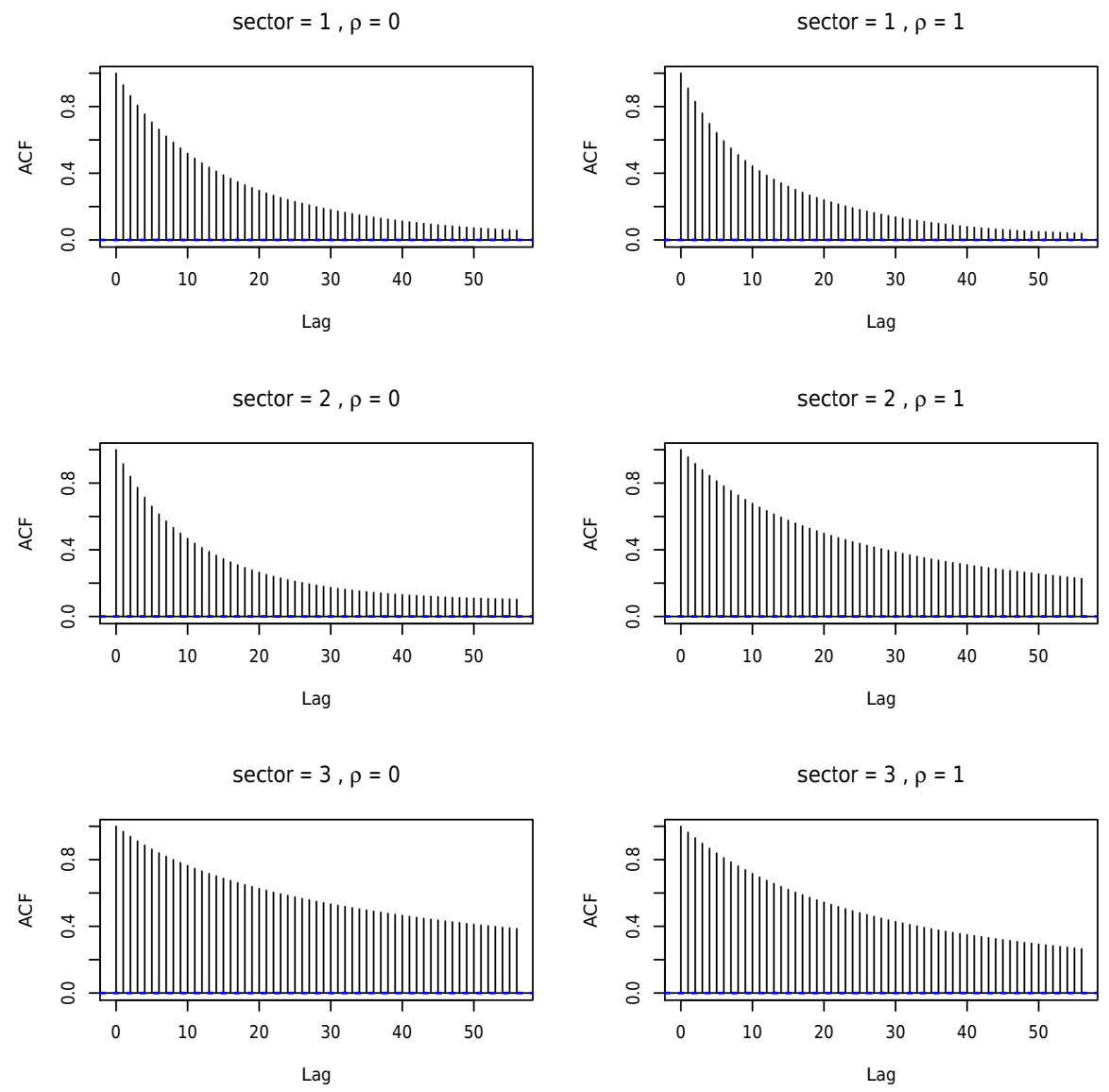

Figure 16: Auto-correlation function of draws for $R$ and the US. 

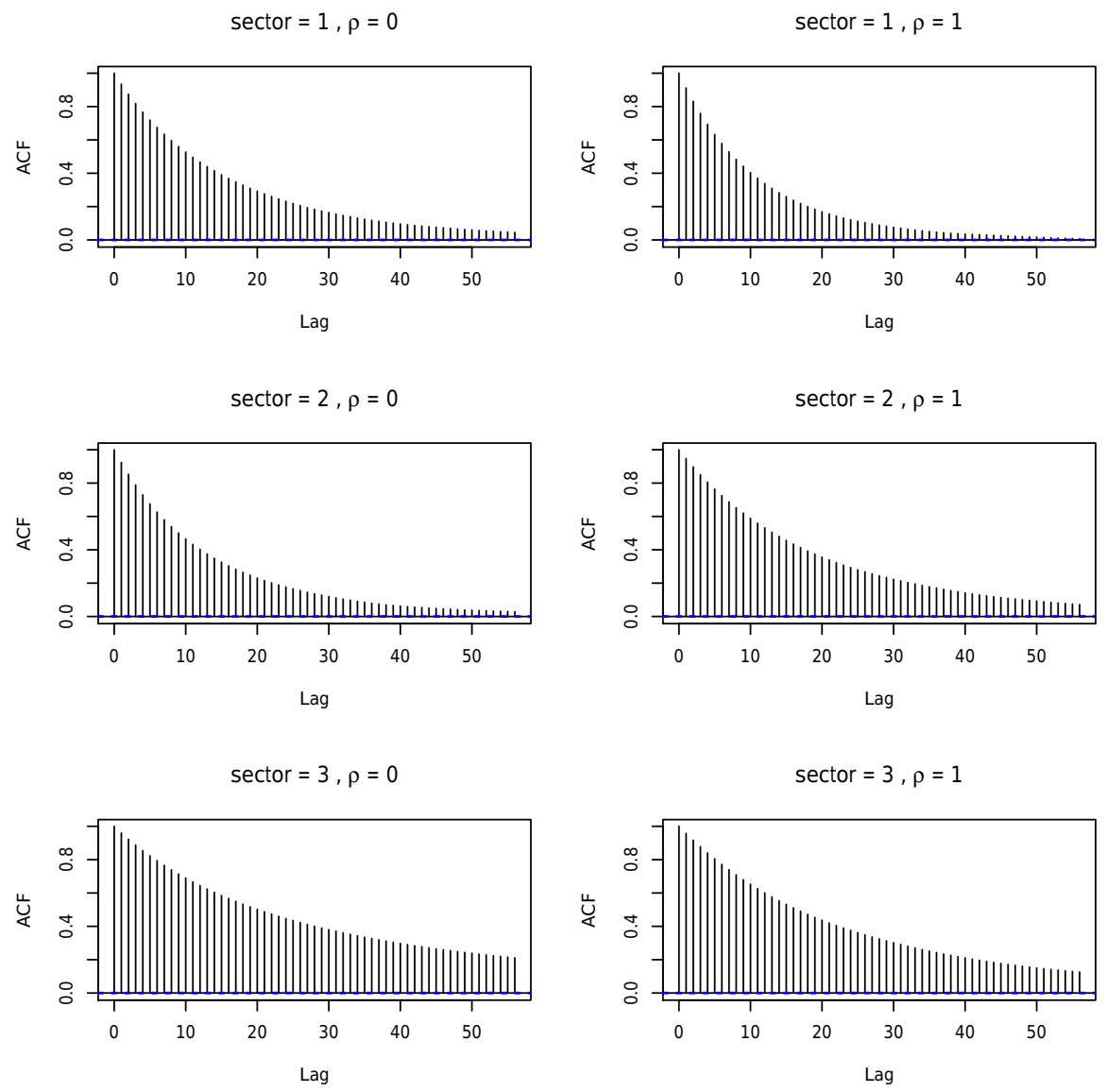

Figure 17: Auto-correlation function of draws for $\hat{\Gamma}$ and the US. 

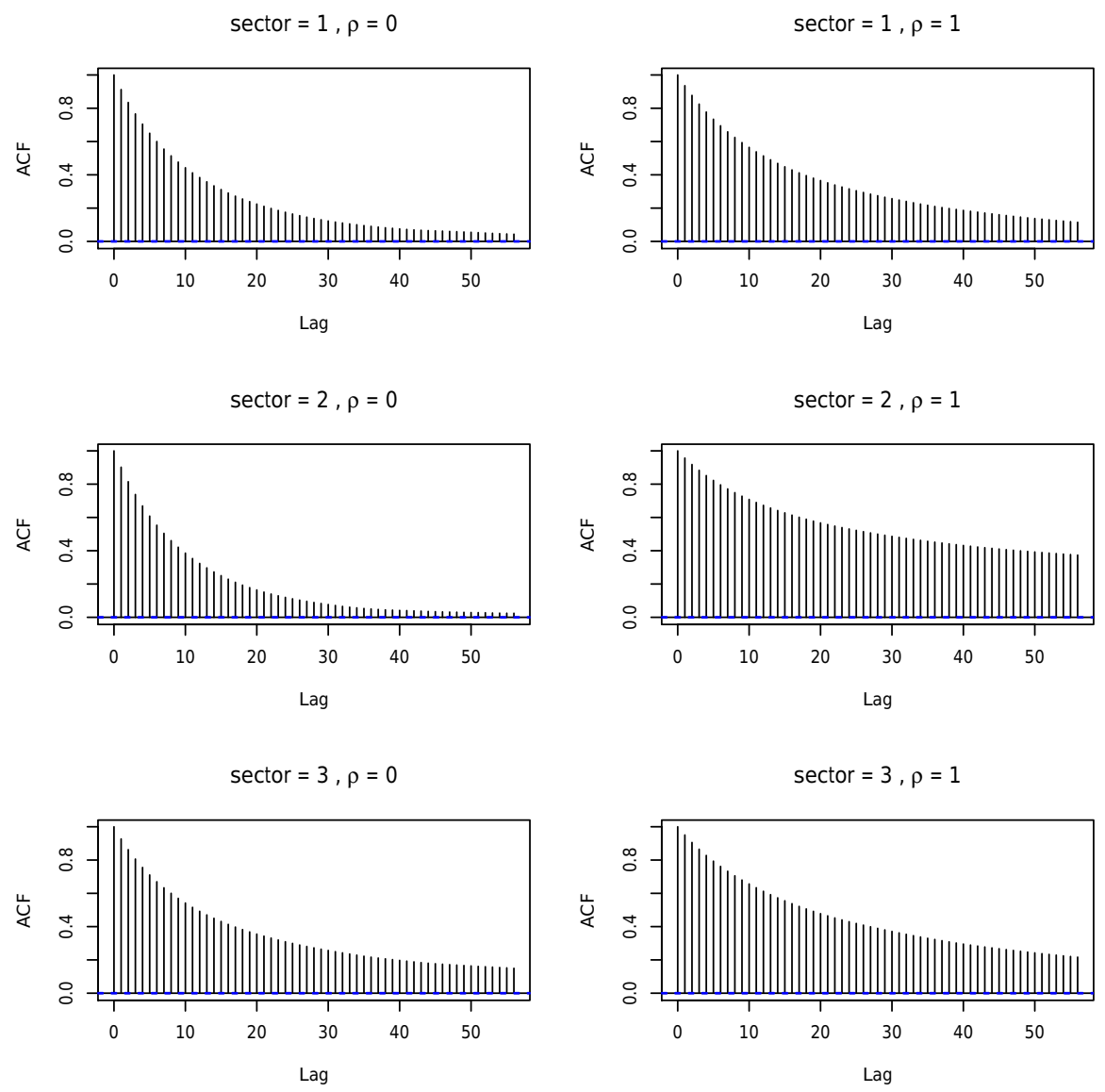

Figure 18: Auto-correlation function of draws for $\bar{\lambda}$ and Japan. 

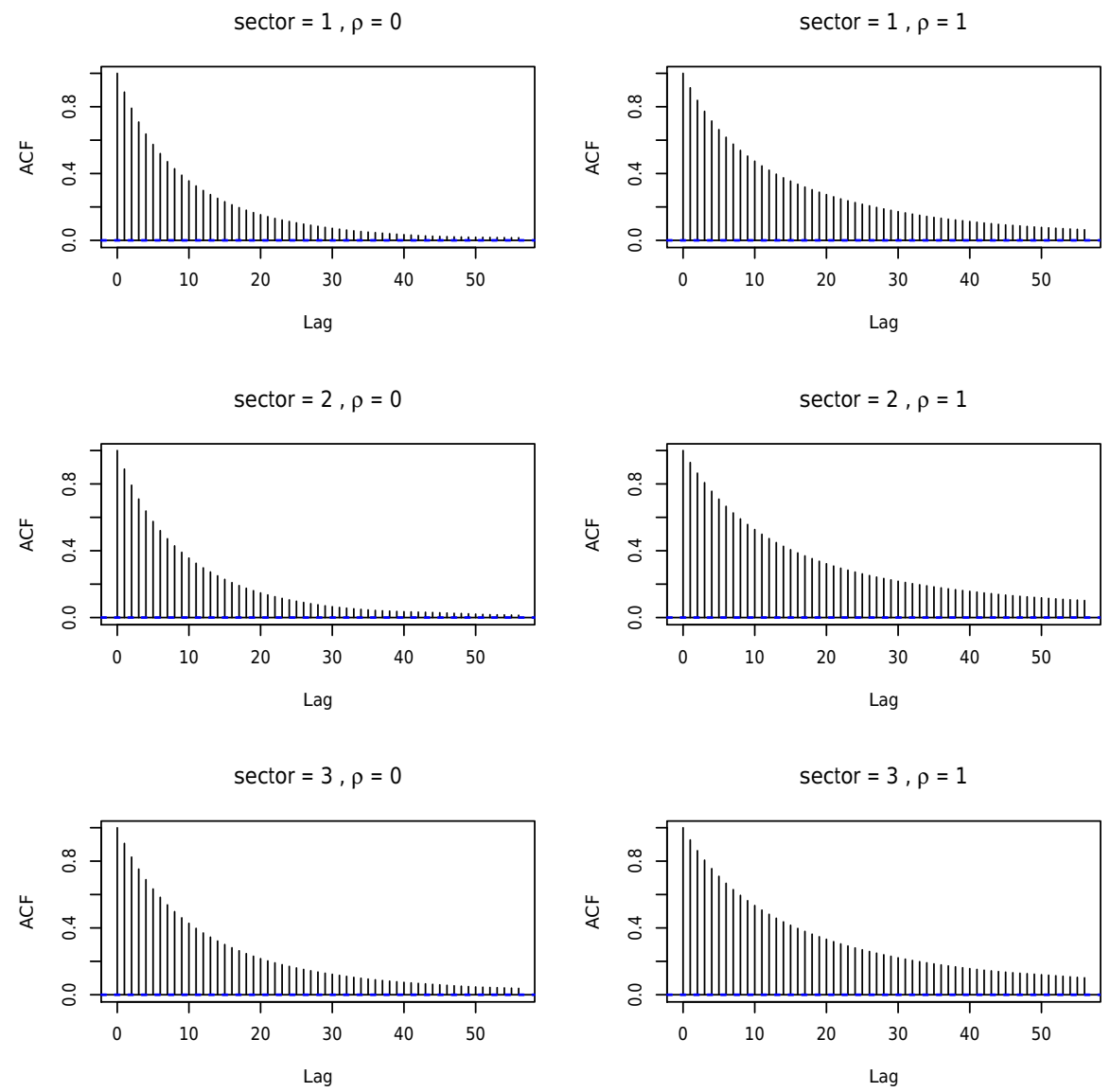

Figure 19: Auto-correlation function of draws for $r$ and Japan. 

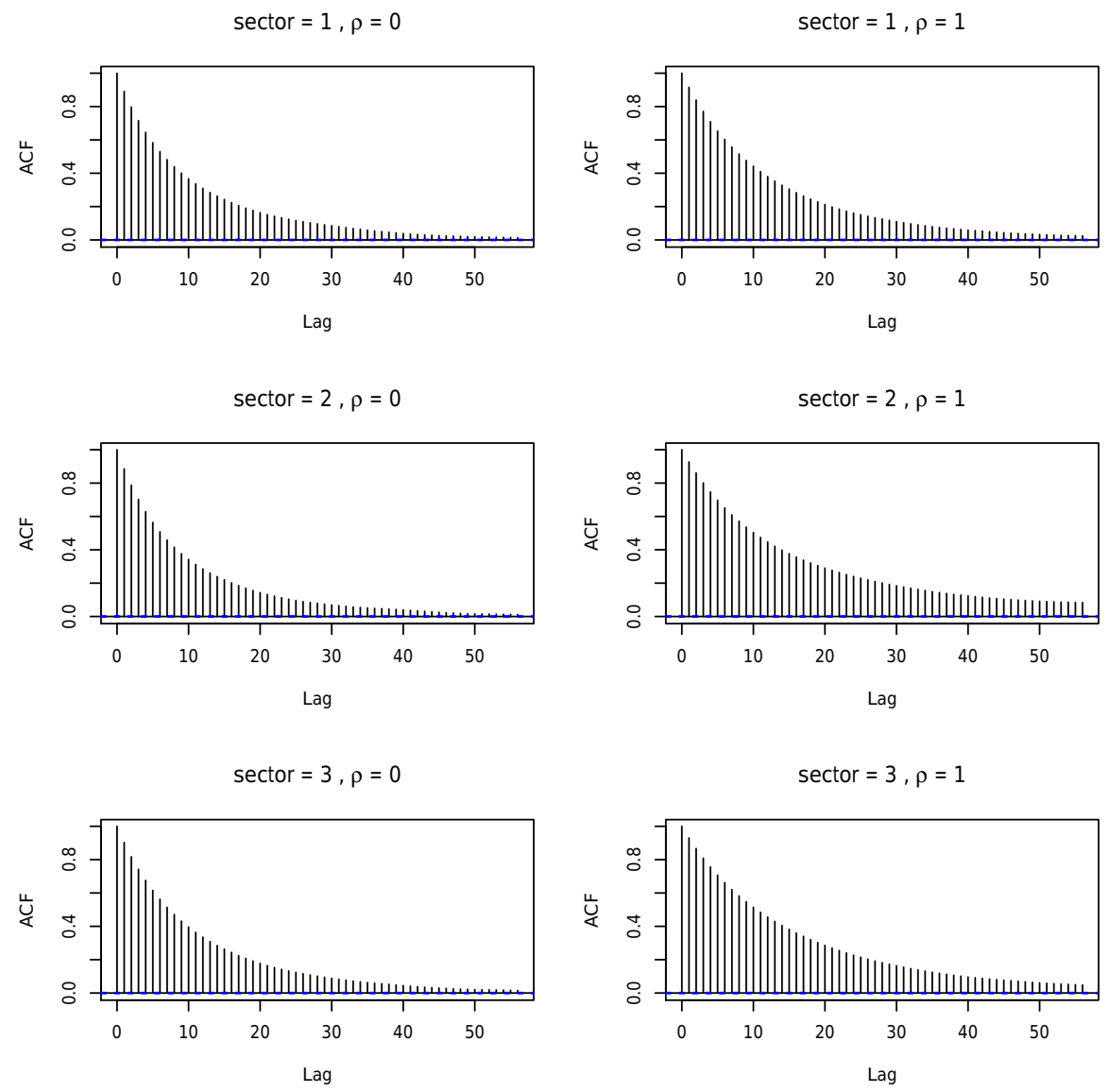

Figure 20: Auto-correlation function of draws for $R$ and Japan. 

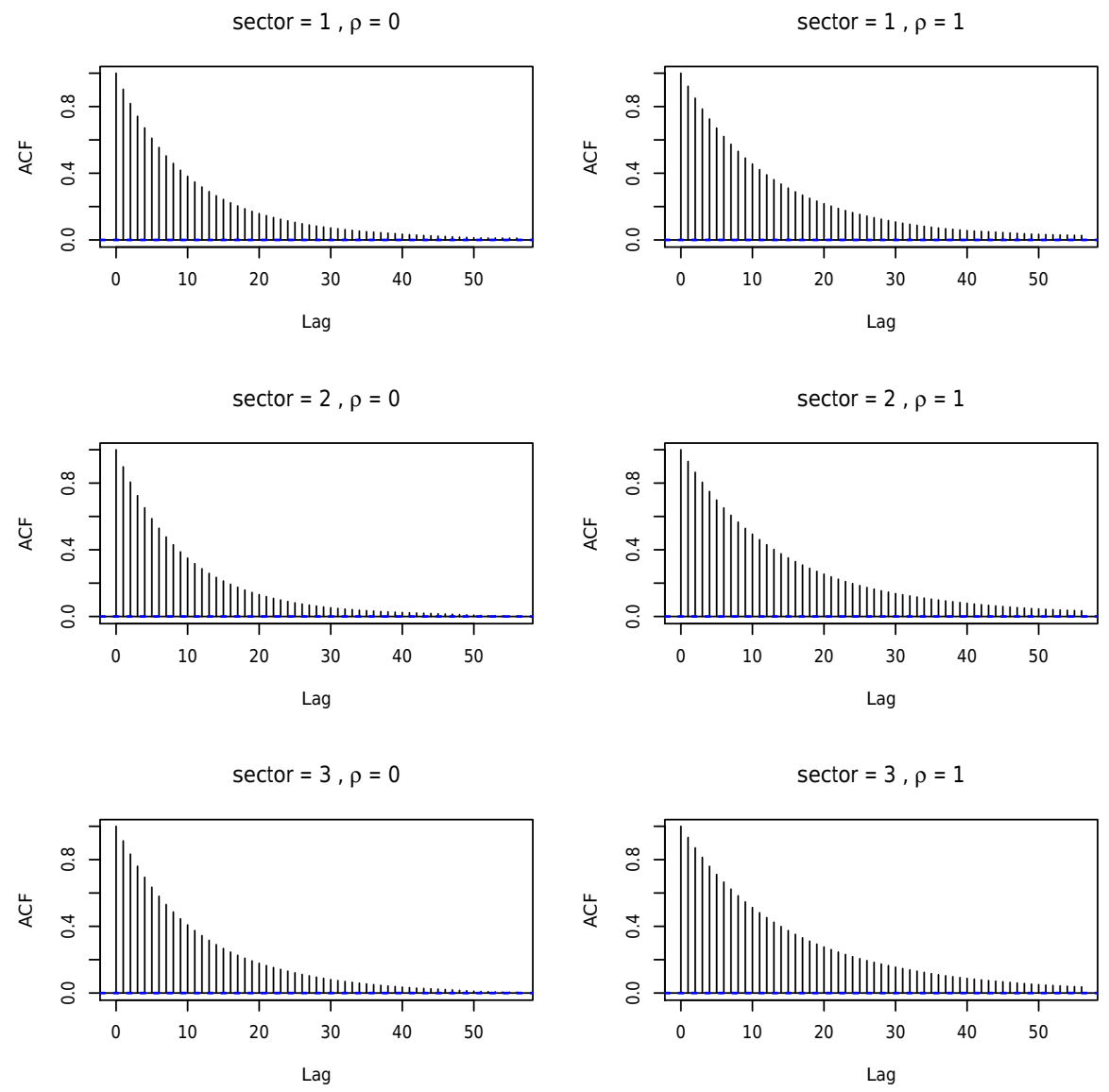

Figure 21: Auto-correlation function of draws for $\hat{\Gamma}$ and Japan. 

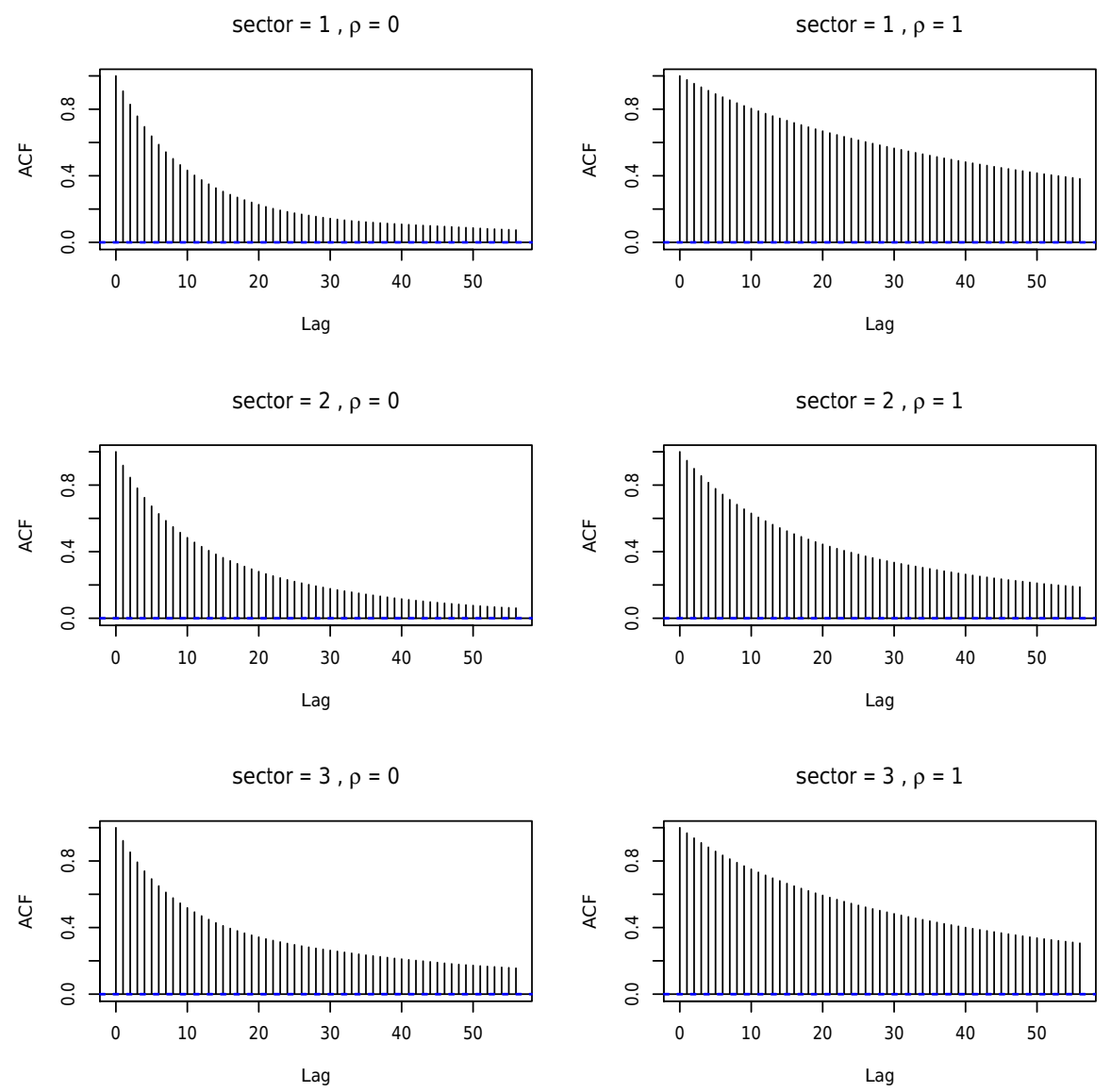

Figure 22: Auto-correlation function of draws for $\bar{\lambda}$ and the UK. 

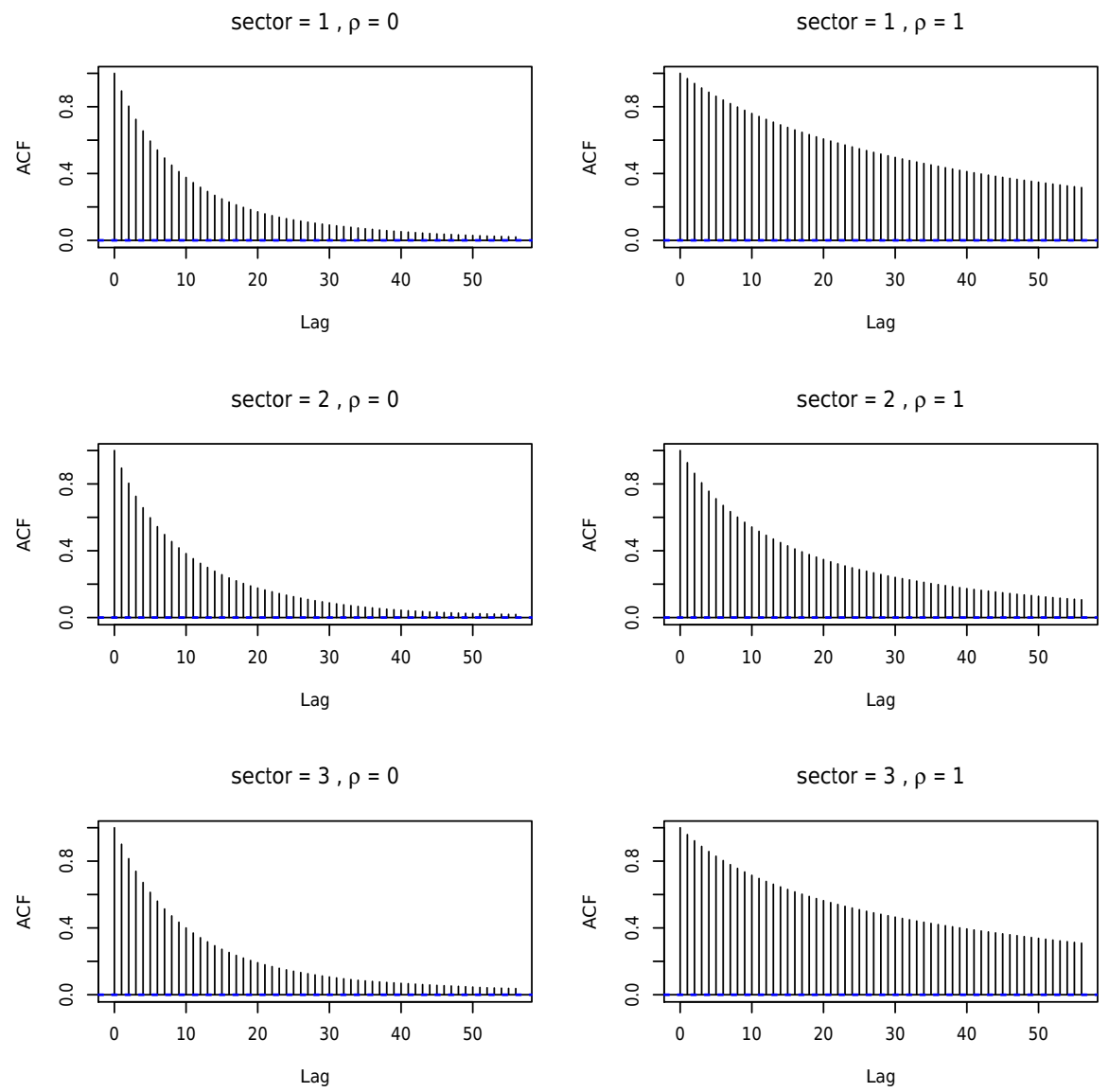

Figure 23: Auto-correlation function of draws for $r$ and the UK. 

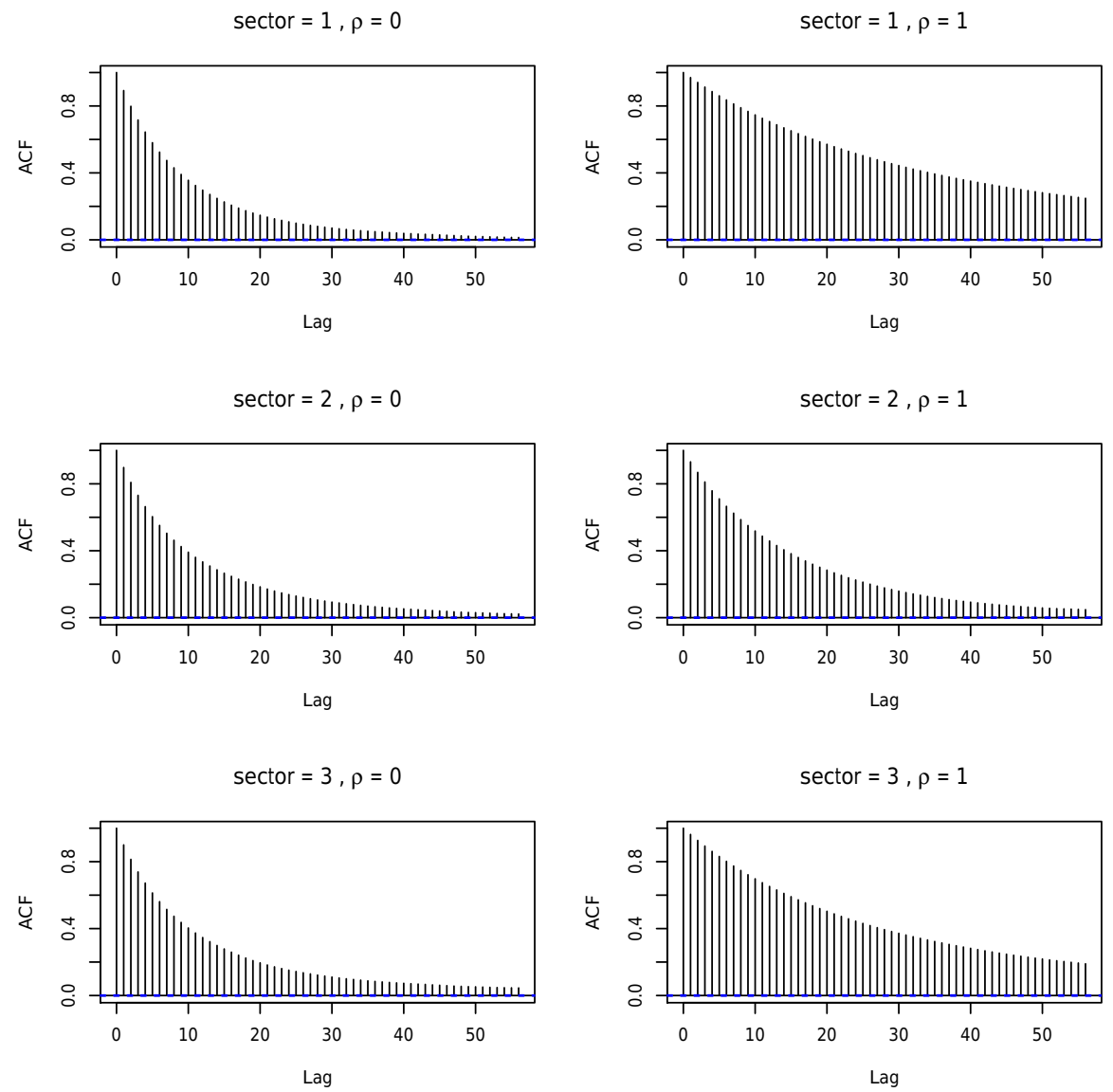

Figure 24: Auto-correlation function of draws for $R$ and the UK. 

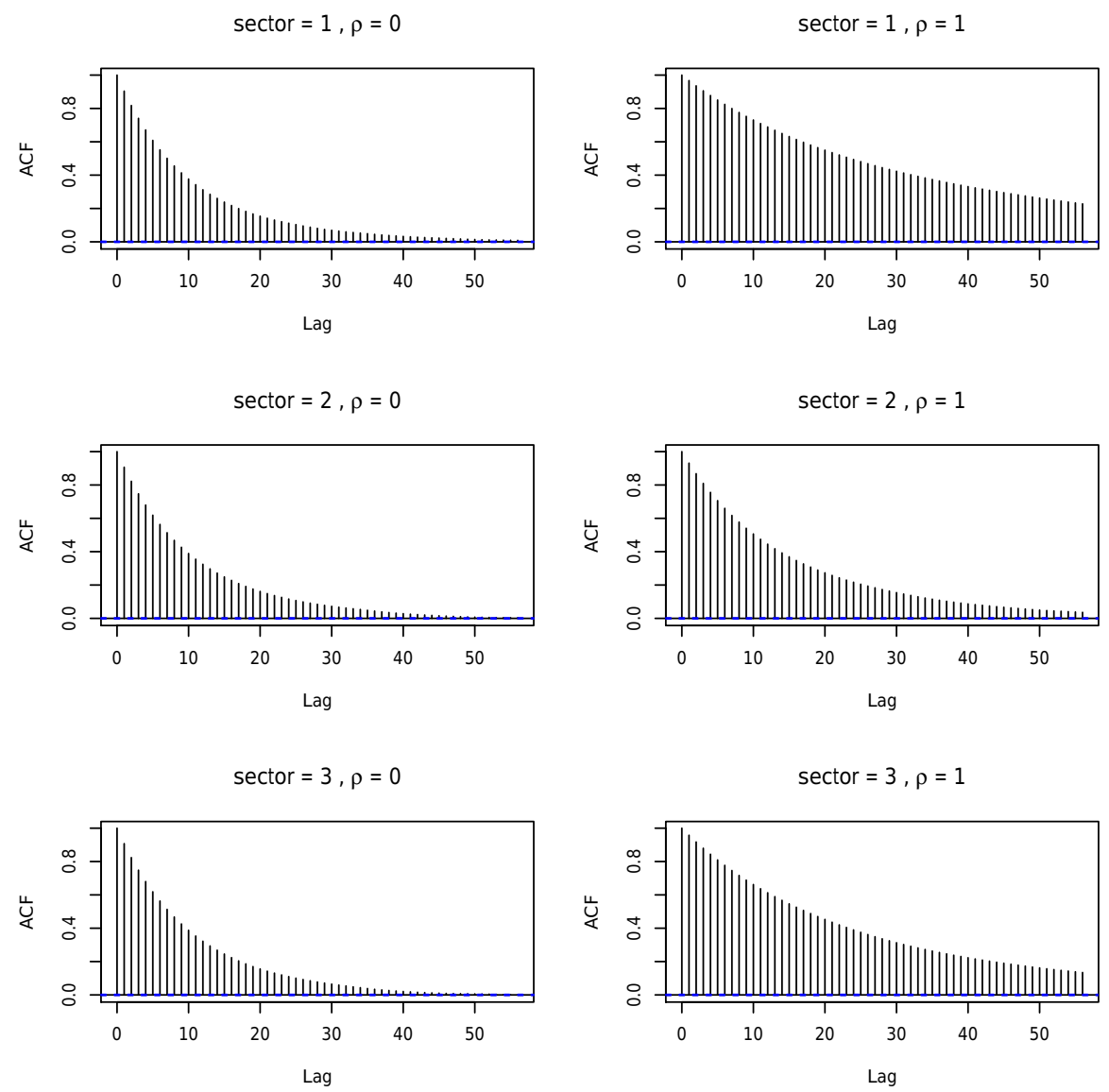

Figure 25: Auto-correlation function of draws for $\hat{\Gamma}$ and the UK. 